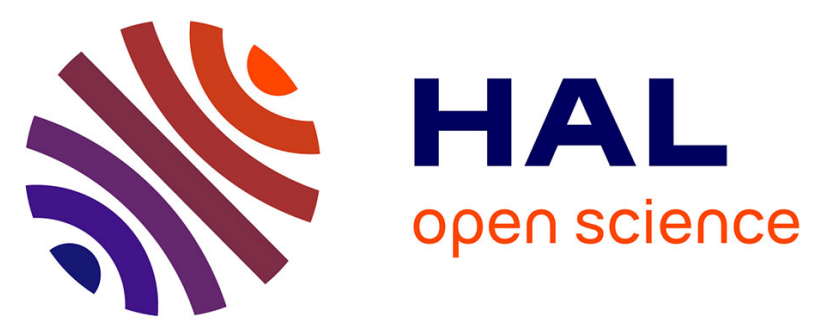

\title{
Anaerobic Membrane BioReactor modeling in the presence of Soluble Microbial Products (SMP) - the Anaerobic Model AM2b
}

Boumediene Benyahia, Tewfik Sari, Brahim Cherki, Jérôme Harmand

\section{To cite this version:}

Boumediene Benyahia, Tewfik Sari, Brahim Cherki, Jérôme Harmand. Anaerobic Membrane BioReactor modeling in the presence of Soluble Microbial Products (SMP) - the Anaerobic Model AM2b. Chemical Engineering Journal, 2013, 228, pp.1011-1022. 10.1016/j.cej.2013.05.073 • hal-00859541

HAL Id: hal-00859541

https://hal.inria.fr/hal-00859541

Submitted on 16 May 2020

HAL is a multi-disciplinary open access archive for the deposit and dissemination of scientific research documents, whether they are published or not. The documents may come from teaching and research institutions in France or abroad, or from public or private research centers.
L'archive ouverte pluridisciplinaire HAL, est destinée au dépôt et à la diffusion de documents scientifiques de niveau recherche, publiés ou non, émanant des établissements d'enseignement et de recherche français ou étrangers, des laboratoires publics ou privés.

\section{(ㅇ)(1) $\$$}

Distributed under a Creative Commons Attribution - NonCommercial - NoDerivatives 44.0 


\title{
Anaerobic Membrane BioReactor modeling in the presence of Soluble Microbial Products (SMP) - the Anaerobic Model AM2b
}

\author{
B. Benyahia ${ }^{\mathrm{a}, \mathrm{b}, *}$, T. Sari ${ }^{\mathrm{b}, \mathrm{c}}$, B. Cherki ${ }^{\mathrm{a}}$, J. Harmand $\mathrm{H}^{\mathrm{b}, \mathrm{d}}$ \\ ${ }^{a}$ University of Tlemcen, Automatics Laboratory, BP 119 Tlemcen 13000, Algeria \\ ${ }^{b}$ Project Modemic, Inra/Inria, UMR Mistea, 2 place Pierre Viala 34060 Montpellier, \\ France \\ ${ }^{c}$ Irstea, UMR Itap, 361 rue Jean-François Breton, 34196 Montpellier, France \\ ${ }^{d}$ INRA, UR050, Laboratory of Environmental Biotechnology, Avenue des Etangs, \\ Narbonne F-11100, France
}

\section{Abstract}

In this paper, we develop a mathematical model of anaerobic membrane bioreactors (AnMBR) for control design purposes. In particular, we integrate into this model the production and the degradation of Soluble Microbial Products (SMP), which are known to play an important role in the membrane fouling phenomenon. The proposed model, named AM2b, is based on the modification of the AM2 two step model initially proposed in [1]. We present a graph-based approach to determinate its equilibria and discuss three different generic cases. We show that under general assumptions (case A), the model developed has the same number of equilibria as the AM2 model and operates in bi-stability [2]. However, under certain operating conditions or if

\footnotetext{
ॠThis work was supported by AiresSud (project 7015), Coadvise (project FP7PEOPLE-IRSES-2008 No. 230833) and Treasure (an Inria Euromed $3+3$ project, http: //project.inria.fr/treasure/). This article is an extension of the IFAC 2011 proceeding [3].

${ }^{*}$ Corresponding author. Tel.: +33(0)4 996124 22; fax: +33(0)46752 1427

Email address: benyahia@supagro.inra.fr (B. Benyahia)
} 
biological parameters values are slightly modified (cases B and C), the AM2b model exhibits equilibria bifurcation and multi-stability property. It is also shown that the AM2b model can provide interesting qualitative information to the user, such as the influence on some equilibria of the variation in the concentration of organic matter in the influent concentration.

Keywords: Anaerobic digestion; Equilibria bifurcation; Membrane fouling; MBR; SMP Modeling; Wastewater treatment plant.

\section{Introduction}

The objectives of a wastewater treatment plant (WWTP) are to reduce the volume of pollutants in discharges, take advantage of the energy that can potentially be produced (such as $\mathrm{CH}_{4}$ in anaerobic treatment) and provide purified effluents for recycling in agriculture and/or industry. The technology of Anaerobic Membrane BioReactors (AnMBR) appears promising for achieving these aims. However, the risk of membrane fouling limits the development of these systems. The use of models coupling the fouling phenomena together with biotic characteristics should enable such fouling to be more predictable and thus, by means of a control, to act in order to limit their impact on system performance.

Among the soluble organic compounds in WWTP effluent, it has been shown that the Soluble Microbial Products (SMP) play a crucial role in the membrane fouling (pore blocking) and their presence in a MBR affects the process performance (effluent quality). It is therefore essential to integrate the SMP into the modeling of bioprocesses.

Most efforts to couple biotic phenomena with membrane models have been 
primarily directed at improving aerobic systems. They have been based on the modification of activated sludge models (ASM) to include SMP formation and degradation. Concerning anaerobic conditions, in contrast, only a few papers are available [4]. Yet anaerobic digestion systems offer many advantages, including the possible use of the resulting biogas as an energy source. Usually, two categories of SMP are considered: BAP (Biomass Associated Products), associated with biomass mortality, and UAP (Utilization Associated Products), associated with substrate degradation and the growth of biomass [5].

In their review [5], Barker and Stuckey noted that the first model to characterize only the SMP formation in a fermentation system was proposed in 1959 by Luedeking and Piret [6]. Later on, this incomplete model was further refined. But the first model to predict both the production and the degradation of SMP in an anaerobic chemostat was proposed by [7], in considering the two categories of SMP (UAP and BAP). It integrated the UAP production but starting from the initial organic matter alone and considered only their degradation by acidogenic bacteria. The BAP were considered to be produced by the decay of acidogenic bacteria and also slowly degraded by them. The model did not take into account either the UAP or BAP production starting from the intermediate product of the reaction (a two step model was considered), nor the decay of methanogenic biomass.

This model was further developed by [8] who have added: i) the concept of UAP production in the consumption of both the initial organic matter and the intermediate products by acidogenic and methanogenic bacteria respectively; ii) BAP production starting from the decay of acidogenic and 
methanogenic bacteria; and iii) BAP degradation by acidogens which grow and give intermediate products.

Later on, [9] proposed a unified theory for the production and the degradation of the UAP and the BAP by envisaging the combination of three types of compound: Extracellular Polymeric Substances (EPS), SMP and inert biomass. They organized their theory through six hypothetical interactions between SMP, EPS, active and inert biomass.

Recently, Aquino and Stuckey [10] in order to predict SMP production, have refined the model suggested in [8] by taking into account the concept of EPS formation and degradation as suggested in [9].

It should be stressed that the majority of the studies cited above relate to conventional systems for anaerobic digestion (chemostat-like systems) and not to membrane reactors. In addition, the most recently developed models are rather complex and clearly unsuited for observer synthesis and/or control system design.

This paper proposes a model which includes the formation and degradation of SMP in an AnMBR, and should prove simple enough to be used for control purposes while capturing the main features related to SMP dynamics. To this end, we propose the AM2b model which we study equilibria and their bifurcations.

This paper is structured as follows: first, we present the model with SMP. Its equilibria are then determined for different generic cases using a graphbased approach. Finally, simulation results are presented which point out different qualitative behavior according to model parameters values. These simulations are discussed before conclusions and perspectives are drawn. 


\section{Development of AM2b model}

\subsection{Hypotheses}

The compartments included in the model are represented in Fig. 1. The process considered here is a side-stream MBR, where $S_{1}$ is the organic matter concentration (COD), $S_{1 \text { in }}$ the input concentration, $S_{2}$ the Volatile Fatty Acid concentration (VFA), $S_{2 i n}$ the input concentration, $X_{1}$ the acidogenic biomass, $X_{2}$ the methanogenic biomass and $S=U A P+B A P$ i.e. the Soluble Microbial Products (SMP) concentration.

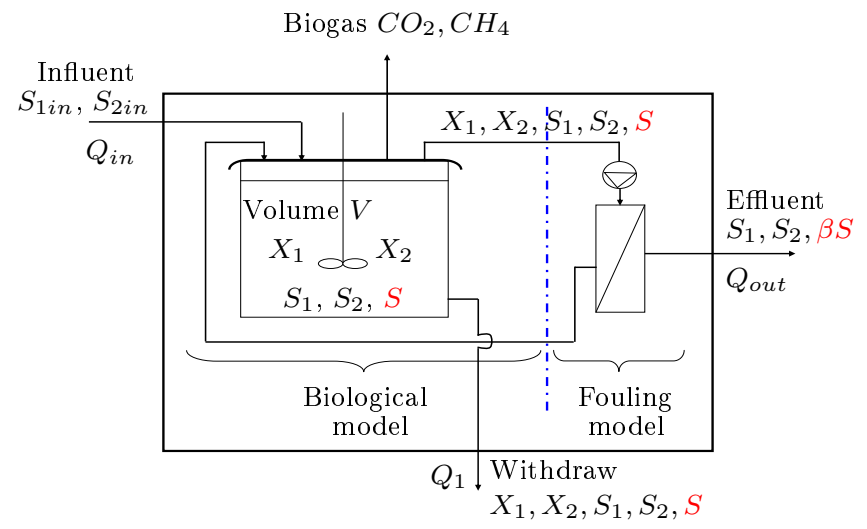

Figure 1: Schematic representation of the proposed model.

A full model of an AnMBR should integrate a biological model of bioreactions taking place in the reaction medium along with a membrane fouling model. In this paper, we develop only the biological model under the following assumptions:

H1. The reaction medium of the bioreactor is considered to be homogeneous, 
H2. The substrates $S_{1}$ and $S_{2}$ go through the external membrane without retention (the size of their molecules is assumed to be smaller than pore diameter),

H3. Total retention of biomasses $X_{1}$ and $X_{2}$ by the membrane is considered (the size of bacteria is greater than pore diameter). Therefore, there are no solids in the effluent and thus no term $-D X_{i}$ in the mass balance equation of $X_{i}$ (see equations (8) and (10)),

H4. Decay rates of biomasses noted $D_{0}$ are taken into account,

H5. The biomass withdrawal with a flowrate $Q_{1}$ is considered,

H6. $S M P=U A P+B A P$ are grouped into a single variable of concentration $S$, of which only a fraction $\beta$ leaves the bioreactor. The remainder, corresponding to macromolecules, is retained by the membrane. This is modeled in the mass balance equation by $-\beta D S$ (see equation (11)), where $0 \leq \beta \leq 1$ ( $\beta=0$ stands for the total retention of SMP by the membrane while $\beta=1$ means free crossing of SMP through the membrane).

On the left of Fig. 2 is represented the separation of matter by the membrane which is formalized through assumptions H2, H3 and H6. On the right is shown the linear working zone of the bioreactor, when the membrane flow $J$ evolves proportionally to the transmembrane pressure $\Delta P$.

\subsection{Reaction network and mathematical model}

Our model is based on the modification of the two-step model AM2 proposed in [1] that has been analyzed by [11] in a chemostat and by [2] in a 

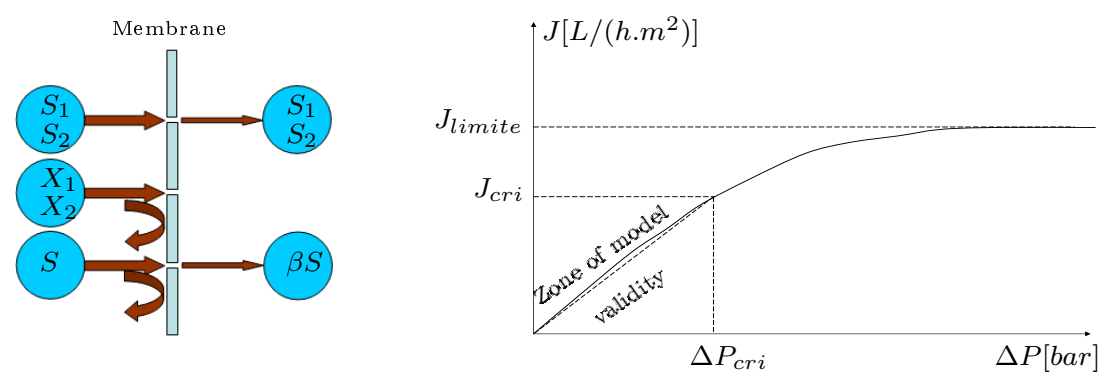

Figure 2: Left: Separation of matter by the membrane. Right: Flux variation depending on transmembrane pressure.

more generic case. In such models, anaerobic digestion is considered as a two-step process:

- first, acidogenesis in which a consortium of acidogenic bacteria $X_{1}$ consume the organic substrate $S_{1}$ and produce $\mathrm{CO}_{2}$ and $S_{2}$ (VFA):

$$
k_{1} S_{1} \stackrel{r_{1}}{\rightarrow} X_{1}+k_{2} S_{2}+k_{4} C O_{2} .
$$

- second, methanogenesis where a consortium of methanogenic bacteria $X_{2}$ use $S_{2}$ as substrate to grow and then produce biogas composed of $\mathrm{CO}_{2}$ and $\mathrm{CH}_{4}$ :

$$
k_{3} S_{2} \stackrel{r_{2}}{\longrightarrow} \mathrm{X}_{2}+k_{5} \mathrm{CO}_{2}+k_{6} \mathrm{CH}_{4} .
$$

We proved in [2] that the AM2 model has at most six equilibrium points and that it can exhibit a bistability. Here, we propose to modify the reaction network (1)-(2) by adding a new compound SMP, named $S$. We model the SMP production from the degradation of $S_{1}, S_{2}$ and the decay of biomasses 
$X_{1}, X_{2}$. In addition, we consider the SMP degradation into $\mathrm{S}_{2}$ and $\mathrm{CO}_{2}$ through the growth of $X_{1}$. The corresponding reaction networks are given as follows:

- Acidogenesis + SMP production:

$$
k_{1} S_{1} \stackrel{r_{1}}{\rightarrow} X_{1}+k_{2} S_{2}+b_{3} S+k_{4} C O_{2}
$$

with the reaction rate: $r_{1}=\mu_{1}\left(S_{1}\right) X_{1}$.

- Methanogenesis + SMP production:

$$
k_{3} S_{2} \stackrel{r_{2}}{\rightarrow} \mathrm{X}_{2}+b_{4} \mathrm{~S}+k_{5} \mathrm{CO}_{2}+k_{6} \mathrm{CH}_{4},
$$

with the reaction rate: $r_{2}=\mu_{2}\left(S_{2}\right) X_{2}$.

- SMP degradation:

$$
b_{1} S \stackrel{r}{\rightarrow} X_{1}+b_{2} S_{2}+k_{7} C O_{2}
$$

with the reaction rate: $r=\mu(S) X_{1}$.

- SMP production from biomass decay:

$$
D_{0} X_{1} \longrightarrow D_{0} S, \quad D_{0} X_{2} \longrightarrow D_{0} S
$$

Let us note $\xi=\left[S_{1}, X_{1}, S_{2}, X_{2}, S\right]^{T}$ the state space vector of the model AM2+SMP (henceforth, this model is named "AM2b"). From the reaction network (3)-(6) and taking into account the above assumptions (H1-H6), the 
law of the conservation of matter enables us to write the following mathematical model:

$$
\begin{aligned}
\frac{d S_{1}}{d t} & =D\left(S_{1 i n}-S_{1}\right)-k_{1} \mu_{1}\left(S_{1}\right) X_{1} \\
\frac{d X_{1}}{d t} & =\left(\mu_{1}\left(S_{1}\right)+\mu(S)-D_{0}-D_{1}\right) X_{1} \\
\frac{d S_{2}}{d t} & =D\left(S_{2 i n}-S_{2}\right)-k_{3} \mu_{2}\left(S_{2}\right) X_{2}+\left(k_{2} \mu_{1}\left(S_{1}\right)+b_{2} \mu(S)\right) X_{1} \\
\frac{d X_{1}}{d t} & =\left(\mu_{2}\left(S_{2}\right)-D_{0}-D_{1}\right) X_{2} \\
\frac{d S}{d t} & =\left(b_{3} \mu_{1}\left(S_{1}\right)+D_{0}-b_{1} \mu(S)\right) X_{1}+\left(b_{4} \mu_{2}\left(S_{2}\right)+D_{0}\right) X_{2}-M S
\end{aligned}
$$

with:

$k_{1}$ : degradation rate of $S_{1}$ by $X_{1}$,

$k_{2}$ : production rate of $S_{2}$ by $X_{1}$ from $S_{1}$,

$k_{3}$ : degradation rate of $S_{2}$ by $X_{2}$,

$b_{1}$ : degradation rate of $S$ by $X_{1}$,

$b_{2}$ : production rate of $S_{2}$ by $X_{1}$ from $S$,

$b_{3}$ : production rate of $S$ by $X_{1}$ from $S_{1}$,

$b_{4}$ : production rate of $S$ by $X_{2}$ from $S_{2}$,

$\beta$ : SMP fraction leaving the bioreactor,

$D$ : dilution rate $\left(=Q_{i n} / V\right)$,

$D_{0}$ : decay rate of biomass, 
$D_{1}$ : withdrawal of biomass $\left(=Q_{1} / V\right)$, $M=\left[\beta D+(1-\beta) D_{1}\right]:$ constant.

Remark 1. : Substituting $S=0$ in the equations (7)-(10) gives the four dimensional model AM2 [1].

Certain biological conditions must prevail in the reaction networks (3), (4) and (5):

- over a given period of time, the quantity of biomass (or products) produced is always smaller than the quantity of substrate consumed (see (4) and (5)). Thus, one has:

$$
\begin{aligned}
& k_{3} \geq 1+b_{4}, \\
& b_{1} \geq 1+b_{2} .
\end{aligned}
$$

- the quantity $S_{2}$ of VFA produced from $S_{1}$ is higher than the quantity produced from the SMP (see (3) and (5)):

$$
k_{2}>b_{2}
$$

\section{Equilibria of model}

\subsection{Characterization of equilibria}

The kinetics $\mu_{1}, \mu_{2}$ and $\mu$ are assumed to be dependent on $S_{1}, S_{2}$ and $S$, respectively. The model analysis given in this paper is valid for all kinetics verifying the following qualitative properties whose patterns are represented as graphs in Fig. 3: 
1. $\mu_{1}\left(S_{1}\right)$ and $\mu(S)$ are increasing functions for $S_{1} \geq 0$ and $S \geq 0$ respectively, with $\mu_{1}(0)=\mu(0)=0$, and $\mu_{1}(+\infty)=m_{1}$ and $\mu(+\infty)=m$.

2. $\mu_{2}\left(S_{2}\right)$ is an increasing function for $0 \leq S_{2}<S_{2}^{M}$; it has a maximum $\mu_{2}\left(S_{2}^{M}\right)>0$ for $S_{2}=S_{2}^{M}$ and it is decreasing for $S_{2}>S_{2}^{M}$, with $\mu_{2}(0)=0$ and $\mu_{2}(+\infty)=0$.
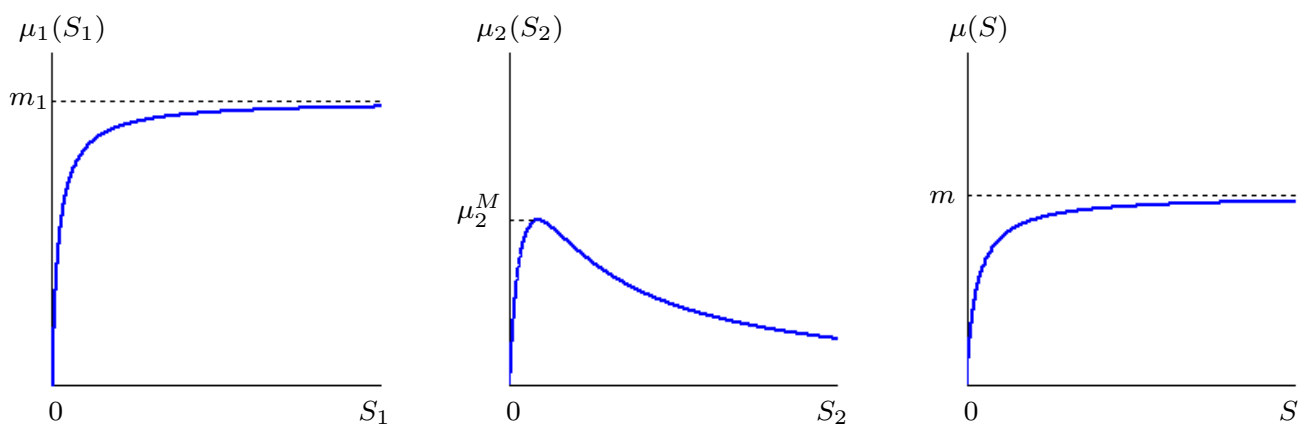

Figure 3: Schematic representation of the kinetic properties.

Examples of such kinetics (used in the sequel) are the Monod functions for $\mu_{1}$ and $\mu$ and the Haldane function for $\mu_{2}$.

$\mu_{1}\left(S_{1}\right)=m_{1} \frac{S_{1}}{S_{1}+K_{1}}, \quad \mu(S)=m \frac{S}{S+K}, \quad \mu_{2}\left(S_{2}\right)=m_{2} \frac{S_{2}}{\frac{S_{2}^{2}}{K_{i}}+S_{2}+K_{2}}$ 
The equilibria of system (7)-(11) are solutions of the following nonlinear algebraic system:

$$
\begin{aligned}
0 & =D\left(S_{1 i n}-S_{1}\right)-k_{1} \mu_{1}\left(S_{1}\right) X_{1} \\
0 & =\left(\mu_{1}\left(S_{1}\right)+\mu(S)-D_{0}-D_{1}\right) X_{1} \\
0 & =D\left(S_{2 i n}-S_{2}\right)-k_{3} \mu_{2}\left(S_{2}\right) X_{2}+\left(k_{2} \mu_{1}\left(S_{1}\right)+b_{2} \mu(S)\right) X_{1} \\
0 & =\left(\mu_{2}\left(S_{2}\right)-D_{0}-D_{1}\right) X_{2} \\
0 & =\left(b_{3} \mu_{1}\left(S_{1}\right)+D_{0}-b_{1} \mu(S)\right) X_{1}+\left(b_{4} \mu_{2}\left(S_{2}\right)+D_{0}\right) X_{2}-M S
\end{aligned}
$$

which is obtained from (7)-(11) by setting the right-hand sides at zero. Three cases are considered:

\section{Case 1. Washout of $X_{1}$ :}

The equilibria $\left(S_{1}^{*}, X_{1}^{*}, S_{2}^{*}, X_{2}^{*}, S^{*}\right)$ of the system (7)-(11) for which $X_{1}^{*}=0$ are given by:

- the washout equilibrium of $X_{1}$ and $X_{2}, E_{0}^{0}=\left(S_{1 i n}, 0, S_{2 i n}, 0,0\right)$, which always exists,

- the washout equilibrium of $X_{1}$ but not of $X_{2}, E_{1}^{i}=\left(S_{1 i n}, 0, S_{2}^{i *}, X_{2}^{i *}, S^{i *}\right)$, $i=1$ or 2 , $X_{2}^{i *}$ and $S^{i *}$ are given by the formulas:

$$
X_{2}^{i *}=\frac{D\left(S_{2 i n}-S_{2}^{i *}\right)}{k_{3}\left(D_{0}+D_{1}\right)}, \quad S^{i *}=\frac{b_{4}+\frac{D_{0}}{D_{0}+D_{1}}}{B k_{3}}\left(S_{2 i n}-S_{2}^{i *}\right), \quad i=1,2
$$


where $S_{2}^{i *}$ are the roots of equation $\mu_{2}\left(S_{2}\right)=D_{0}+D_{1}$ and,

$$
B=\left[\beta+(1-\beta) \frac{D_{1}}{D}\right]=\frac{M}{D}
$$

The equilibrium $E_{1}^{i}$ exists if and only if:

$$
S_{2 i n}>S_{2}^{i *}
$$

\section{Case 2. Washout of $X_{2}$ but not of $X_{1}$ :}

Let $E^{*}=\left(S_{1}^{*}, X_{1}^{*}, S_{2}^{*}, X_{2}^{*}, S^{*}\right)$ an equilibrium point of system (7)-(11). If $X_{1}^{*}>0$ and $X_{2}^{*}=0$ then one has $0<S_{1}^{*}<S_{1 i n}, S_{2}^{*}>0$ and $S^{*}>0$. Moreover $S_{1}^{*}$ and $S^{*}$ are solutions of the system of equations:

$$
\left\{\begin{array}{l}
S_{1}=F_{m}(S) \\
S=G\left(S_{1}\right)
\end{array}\right.
$$

where $F_{m}$ and $G$ are defined by:

$$
\begin{aligned}
F_{m}(S) & :=\mu_{1}^{-1}\left(D_{0}+D_{1}-\mu(S)\right) \\
G\left(S_{1}\right) & :=\left(S_{1 i n}-S_{1}\right)\left(B_{1}+\frac{B_{2}}{\mu_{1}\left(S_{1}\right)}\right)
\end{aligned}
$$

with:

$$
B_{1}=\frac{b_{3}+b_{1}}{k_{1} B}, \quad B_{2}=\frac{D_{0}-b_{1}\left(D_{0}+D_{1}\right)}{k_{1} B} .
$$

$X_{1}^{*}$ and $S_{2}^{*}$ are given by the formulas:

$$
X_{1}^{*}=D \frac{S_{1 i n}-S_{1}^{*}}{k_{1} \mu_{1}\left(S_{1}^{*}\right)}, \quad S_{2}^{*}=S_{2 i n}+\left[k_{2} \mu_{1}\left(S_{1}^{*}\right)+b_{2} \mu\left(S^{*}\right)\right] \frac{S_{1 i n}-S_{1}^{*}}{k_{1} \mu_{1}\left(S_{1}^{*}\right)}
$$


The equilibrium $E^{*}$ exists if and only if:

$$
S_{1 i n}>S_{1}^{*}
$$

\section{Case 3. No washout of $X_{1}$ and $X_{2}$ :}

Let $E^{*}=\left(S_{1}^{*}, X_{1}^{*}, S_{2}^{*}, X_{2}^{*}, S^{*}\right)$ an equilibrium point of system (7)-(11). If $X_{1}^{*}>0$ and $X_{2}^{*}>0$, then one has $0<S_{1}^{*}<S_{1 i n}, S_{2}^{*}=S_{2}^{i *}$, $i=1,2$ and $S^{*}>0$. Moreover, $S_{1}^{*}$ and $S^{*}$ are solutions of the system of equations:

$$
\left\{\begin{array}{l}
S_{1}=F_{m}(S) \\
S=H_{i}\left(S_{1}\right), \quad i=1,2
\end{array}\right.
$$

where $H_{i}, i=1,2$ are defined by:

$$
H_{i}\left(S_{1}\right):=C_{1 i}+\left(S_{1 i n}-S_{1}\right)\left(C_{2}+\frac{C_{3}}{\mu_{1}\left(S_{1}\right)}\right), \quad i=1,2
$$

with:

$$
\begin{gathered}
C_{1 i}=\frac{A}{B}\left(S_{2 i n}-S_{2}^{i *}\right), C_{2}=\frac{A\left(k_{2}-b_{2}\right)+b_{3}+b_{1}}{k_{1} B}, C_{3}=\frac{\left(A b_{2}-b_{1}\right)\left(D_{0}+D_{1}\right)+D_{0}}{k_{1} B}, \\
A=\frac{b_{4}\left(D_{0}+D_{1}\right)+D_{0}}{k_{3}\left(D_{0}+D_{1}\right)} .
\end{gathered}
$$

$X_{1}^{*}$ and $X_{2}^{*}$ are given by the formulas:

$$
X_{1}^{*}=D \frac{S_{1 i n}-S_{1}^{*}}{k_{1} \mu_{1}\left(S_{1}^{*}\right)}, \quad X_{2}^{i *}=D \frac{\left[S_{1 i n}-S_{1}^{*}\right]\left[k_{2} \mu_{1}\left(S_{1}^{*}\right)+b_{2} \mu\left(S^{*}\right)\right]+\left(S_{2 i n}-S_{2}^{i *}\right) k_{1} \mu_{1}\left(S_{1}^{*}\right)}{k_{1} k_{3}\left(D_{0}+D_{1}\right) \mu_{1}\left(S_{1}^{*}\right)}
$$

The equilibrium $E^{*}$ exists if and only if the condition (26) holds to- 
Author-produced version of the article published in Chemical Engineering Journal, 2013, 228, 1011-1022. The original publication is available at http://www.sciencedirect.com.

DOI : 10.1016/j.cej.2013.05.073

gether the following condition:

$$
H_{i}\left(S_{1}^{*}\right)>G\left(S_{1}^{*}\right), \quad i=1,2 .
$$

The solutions of the system (16)-(20) are summarized in Fig. 4:

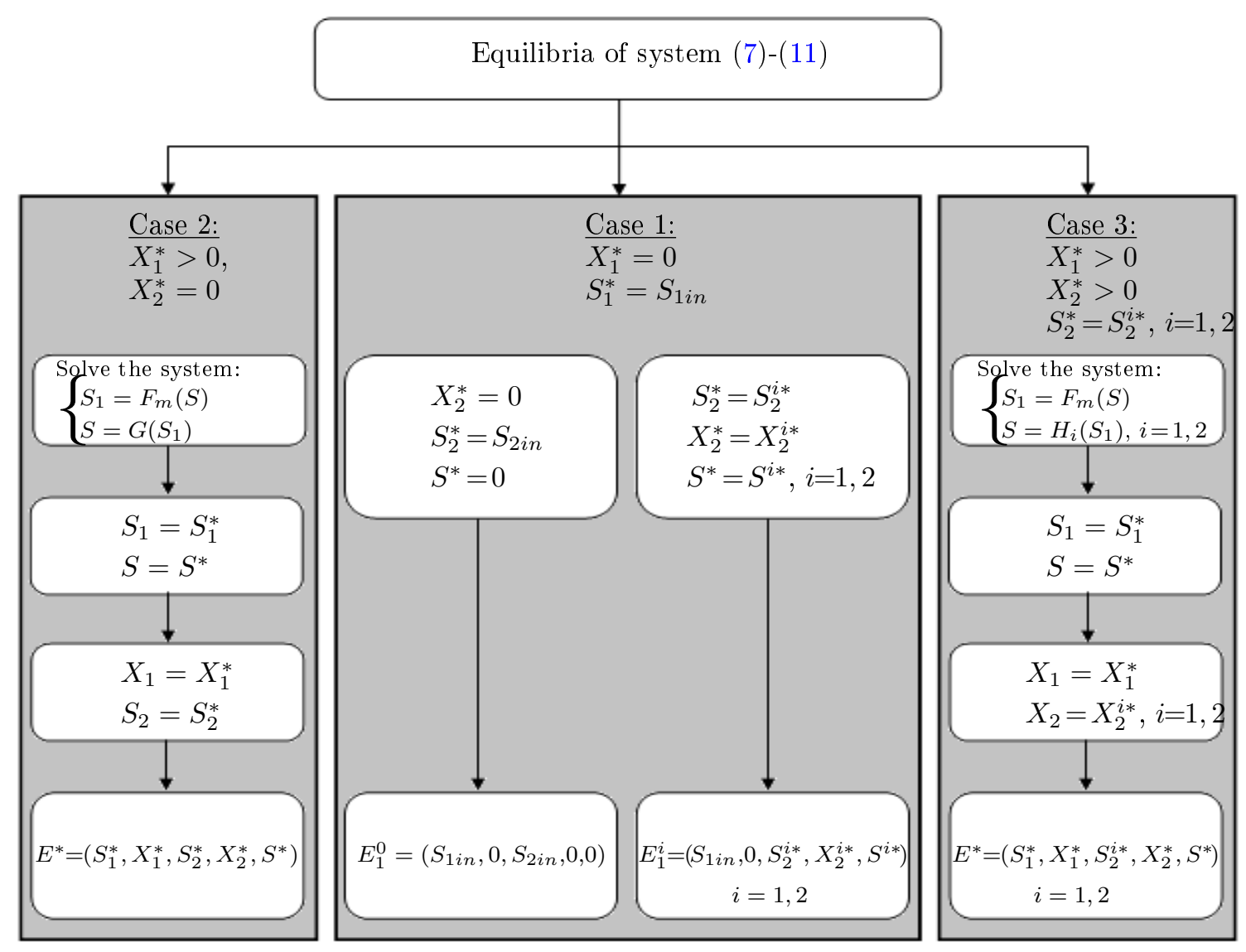

Figure 4: Diagram summarizing the equilibria of system (7)-(11). 


\subsection{Graphical determination of equilibria}

In this section, we propose a graph-based approach to determine the equilibria of system (7)-(11). It should be noted that only the positive values of $S_{1}^{*}$ and $S^{*}$, which verify conditions (26) and (31) are considered.

Using (12) and (13), we show that $B_{2}<0$ and $C_{3}<0$. Hence $G$ and $H_{i}$ tend to $-\infty$ as $S_{1}$ tends to 0 . We assume that $B_{2}>-m_{1} B_{1}, C_{3}>-m_{1} C_{2}$, so that $G$ and $H_{i}$ tend to $-\infty$ as $S_{1}$ tends to $+\infty$. Thus, the functions $G$ and $H_{i}$ are as in Figs. 5, 6 and 7. These figures are obtained with functions (15) and the values for biological parameters given in Table 1 (see below for cases A, B and C).

The parameters of the part of the model part without SMP (original model AM2) have values equal or close to the AMOCO values [1] (by using the standard deviation, see Table III in [1]). The other parameters $b_{1}, b_{2}, b_{3}$ and $b_{4}$ (the parameters of the part modeling the SMP), have values satisfying conditions (12), (13) and (14) and are chosen according to:

- $b_{1}<k_{1}$ : $S$ is slowly degraded in relation to $S_{1}$,

- $b_{3}<k_{2}$ : the quantity of $S_{1}$ transformed in $S_{2}$ is higher than the quantity transformed in $S$,

- $b_{4}<k_{1}, k_{2}, k_{3}$ : the quantity of $S_{2}$ transformed in $S$ is smaller than other quantities produced or degraded.

Units of yield coefficients are in $[g / g]$; concentrations, inhibition and halfsaturation constants are in $[g / l]$; dilution rates, decay rates and maximum growth rates are in $[1 / d]$. 
Table 1: Nominal values for the parameters of the AM2b model

\begin{tabular}{|c|c||c|c||c|c|}
\hline Parameter & Value & Parameter & Value & Parameter & Value \\
\hline$m_{1}$ & 1.2 & $\beta$ & 0.6 & $b_{2}$ & 0.6 \\
$K_{1}$ & 10,16 or 18 & $k_{1}$ & 25 & $b_{3}$ & 7 \\
$m_{2}$ & 1.5 & $k_{2}$ & 15 & $b_{4}$ & 5 \\
$K_{2}$ & 0.3 & $k_{3}$ & 16.08 & $m$ & varying \\
$K_{I}$ & 0.9 & $b_{1}$ & 5 & $K$ & 3 \\
\hline \hline$D$ & 1 & $D_{0}$ & 0.25 & $D_{1}$ & 0.4 or 0.25 \\
$S_{1 i n}$ & 15 or 10 & $S_{2 i n}$ & 1 or 0.6 & & \\
\hline
\end{tabular}

The difference between Figs. 5, 6 and 7 consists in the values of the parameters $K_{1}, S_{1 i n}, S_{2 i n}$ and $D_{1}$. More precisely, we have:

- Generic Case A: $K_{1}=10, S_{1 i n}=15, S_{2 i n}=1$ and $D_{1}=0.4$ (see Fig. $5)$,

- Generic Case B: $K_{1}=16, S_{1 i n}=15, S_{2 i n}=1$ and $D_{1}=0.4$ (see Fig. $6)$,

- Generic Case C: $K_{1}=18, S_{1 i n}=10, S_{2 i n}=0.6$ and $D_{1}=0.25$, (see Fig. 7),

with $\lambda_{1}=\mu_{1}^{-1}\left(D_{0}+D_{1}\right)$.

In all cases, we vary the value of the bifurcation parameter $m$ to change the form of $F_{m}(S)$ since it depends on this parameter of $\mu(S)$ (see $(23)$ ). Since functions $H_{i}\left(S_{1}\right)$ and $G\left(S_{1}\right)$ do not depend on the kinetics $\mu(S)$, their graphs do not change with $m$ (see (24), (28) and Figs. 5, 6 and 7).

Notice that $F_{m}(S)$ can have zero, one or two intersections with $H_{1}\left(S_{1}\right)$, $H_{2}\left(S_{1}\right)$ or $G\left(S_{1}\right)$ (see Figs. 5, 6, and 7). Let us use the following notations for equilibria: 
- $E_{2 j}^{1}$ : equilibria given by the intersection of $F_{m}(S)$ with $H_{1}\left(S_{1}\right), j=1,2$,

- $E_{2 j}^{2}$ : equilibria given by the intersection of $F_{m}(S)$ with $H_{2}\left(S_{1}\right), j=1,2$,

- $E_{2 j}^{0}$ : equilibria given by the intersection of $F_{m}(S)$ with $G\left(S_{1}\right), j=1,2$.

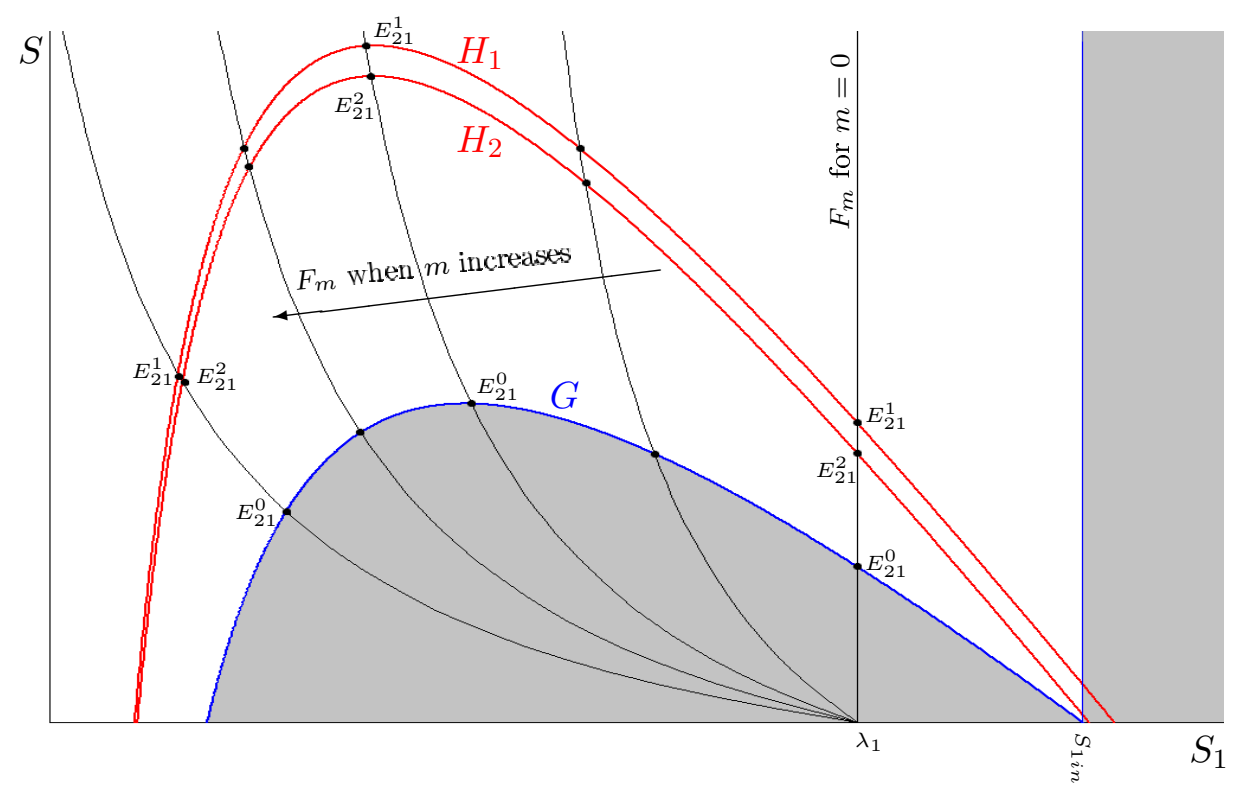

Figure 5: Intersection of the graph of $F_{m}(S)$ with the graphs $G\left(S_{1}\right)$ and $H_{i}\left(S_{1}\right)$ in the Case A.

Areas where the condition (26) and/or the condition (31) are not satisfied have been colored in gray. Therefore, any intersection of $F_{m}(S)$ with $H_{1}\left(S_{1}\right)$ or $H_{2}\left(S_{1}\right)$ which is within these areas is not considered.

\section{Numerical simulations}

In this section, we analyze the three generic cases illustrated by Figs. 5, 6 and 7 . We characterize the number and the nature of equilibria according to the bifurcation parameter $m$. In addition, we highlight the capability of 
Author-produced version of the article published in Chemical Engineering Journal, 2013, 228, 1011-1022. The original publication is available at http://www.sciencedirect.com.

DOI : 10.1016/j.cej.2013.05.073

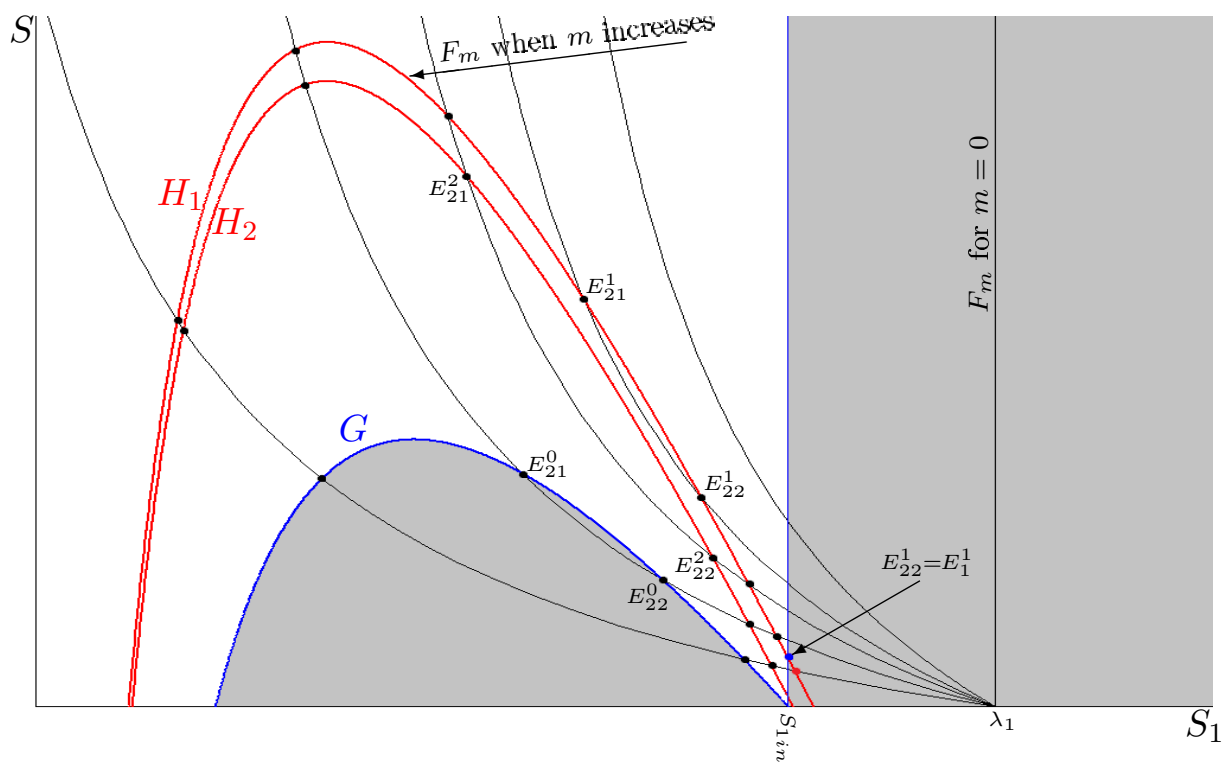

Figure 6: Intersection of the graph of $F_{m}(S)$ with the graphs $G\left(S_{1}\right)$ and $H_{i}\left(S_{1}\right)$ in the Case B.

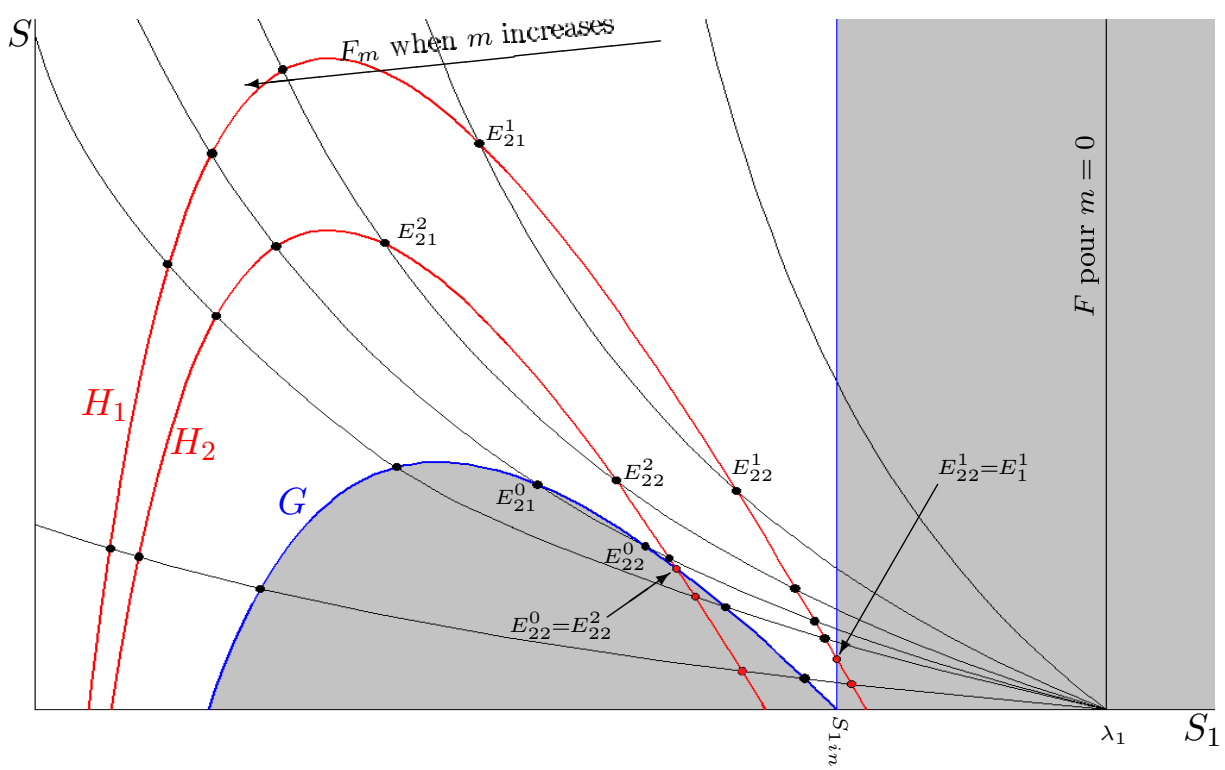

Figure 7: Intersection of the graph of $F_{m}(S)$ with the graphs $G\left(S_{1}\right)$ and $H_{i}\left(S_{1}\right)$ in the Case C. 
the AM2b model to predict a number of qualitative features of interest from a practical point of view.

\subsection{Generic Case A: No equilibria bifurcation of AM2b}

Case A is illustrated in Fig. 5. The intersection of the graph of $F_{m}(S)$ with graphs of $G\left(S_{1}\right), H_{1}\left(S_{1}\right)$ and $H_{2}\left(S_{1}\right)$ give three equilibria $E_{21}^{0}, E_{21}^{1}$ and $E_{21}^{2}$ whatever the value of $m$ (in addition to the trivial equilibria). The system keeps the number and the nature of its equilibria. Only the values of their components change when $m$ varies, as represented in Fig. 9. The trivial equilibria $E_{0}^{0}, E_{1}^{1}$ and $E_{1}^{2}$ are represented in Fig. 8: they exist if and only if (21) holds and they do not depend on the parameter $m$. Here, we have:

$$
S_{2}^{1 *}=0.3123<S_{2}^{2 *}=0.8647<S_{2 i n}=1
$$

In total, the system can have six equilibria, summarized in Table 2, where T stands for Trivial Equilibria, while $F_{m} \cap H_{1}, F_{m} \cap H_{2}$ and $F_{m} \cap G$ stand for Equilibria obtained by the intersections of the graph $F_{m}$ with graphs $H_{1}, H_{2}$ and $G$, respectively, and where $\mathrm{S}$ and $\mathrm{U}$ stand for a Stable and an Unstable Equilibrium, respectively.

Table 2: Equilibria and their nature in the generic case A represented in Fig. 5.

\begin{tabular}{|c||c|c|c|c|c|c|}
\hline \multirow{2}{*}{ case $\mathbf{A}$} & \multicolumn{6}{|c|}{ Equilibria and nature } \\
\cline { 2 - 7 } & $E_{0}^{0}$ & $E_{1}^{1}$ & $E_{1}^{2}$ & $F_{m} \cap G$ & $E_{m} \cap H_{2}$ & $F_{m} \cap H_{1}$ \\
\hline \hline$m \geq 0$ & $\mathrm{U}$ & $\mathrm{U}$ & $\mathrm{U}$ & $\mathrm{S}$ & $E_{21}^{2}$ & $E_{21}^{1}$ \\
\hline
\end{tabular}

According to these results, we conclude that in operating conditions of the generic case $\mathrm{A}$, the original system AM2, modified by the introduction 
of the new variable $S$, keeps the number and the nature of its equilibria and always operates in bistability (cf. [2]). Only the values of $E_{21}^{0}, E_{21}^{1}$ and $E_{21}^{2}$ change with respect to $m$.

$S_{1}$
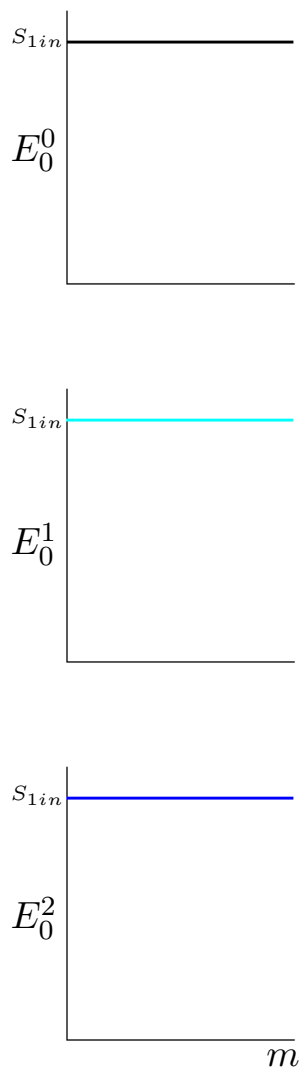

$X_{1}$
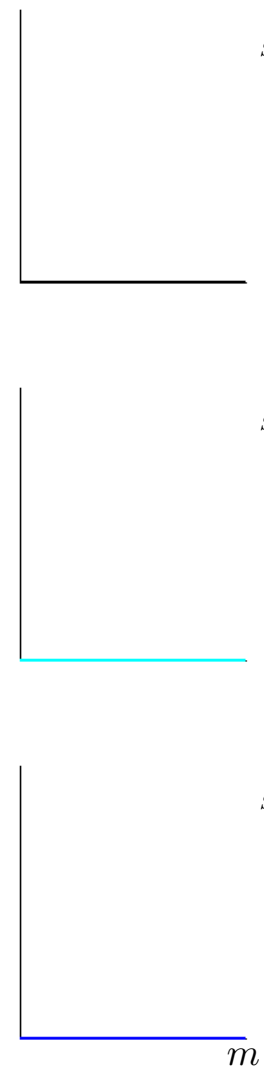

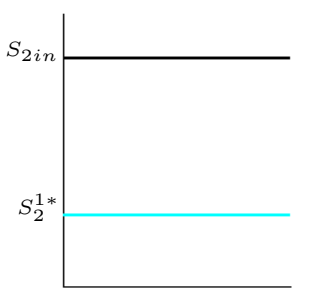

$S_{2}$
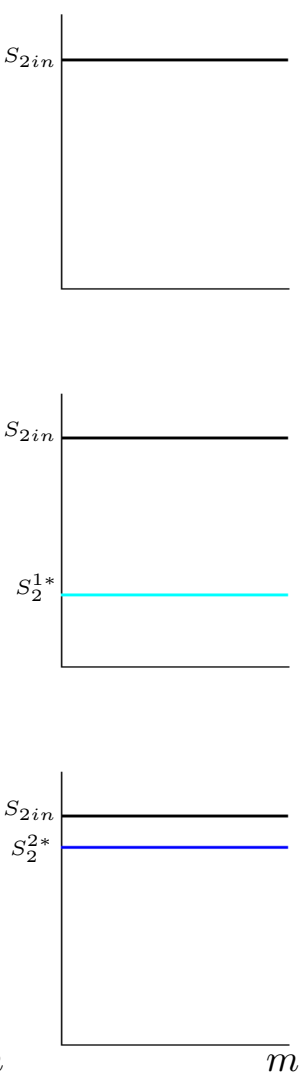

$X_{2}$
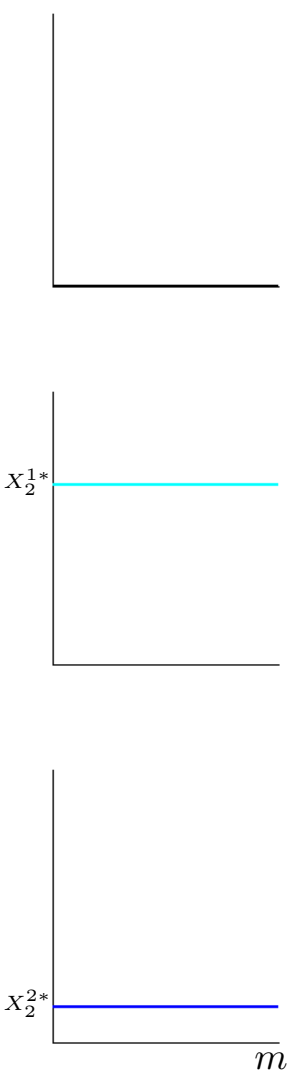

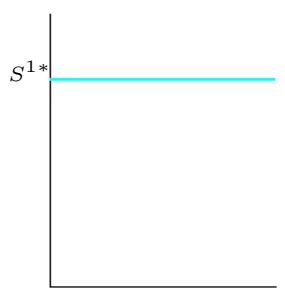

$S$
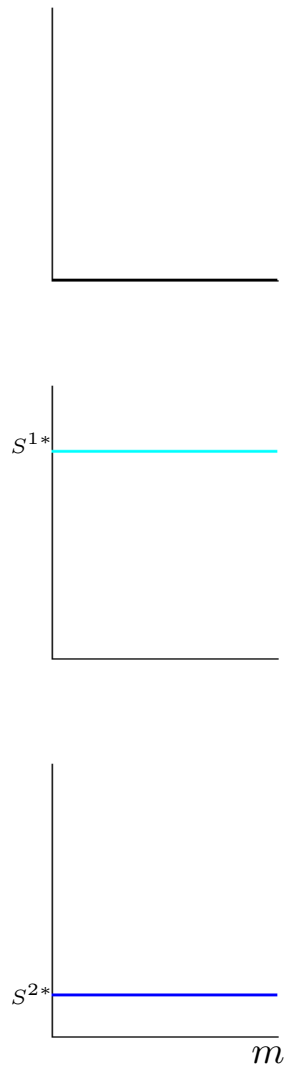

Figure 8: The components of the trivial equilibria $E_{0}^{0}$ (in black), $E_{0}^{1}$ (in cyan) and $E_{0}^{2}$ (in blue) in the Case A.

\subsection{Generic Case B: Equilibria bifurcation for the AM2b, first example}

We represent this case of operating conditions in Fig. 6. For $m=0$, there is no intersection between the graph of $F_{m}(S)$ and the graphs of $G\left(S_{1}\right)$ and $H_{i}\left(S_{1}\right)$. Hence, there are no equilibria except washout equilibria $E_{0}^{0}, E_{1}^{1}$ and 

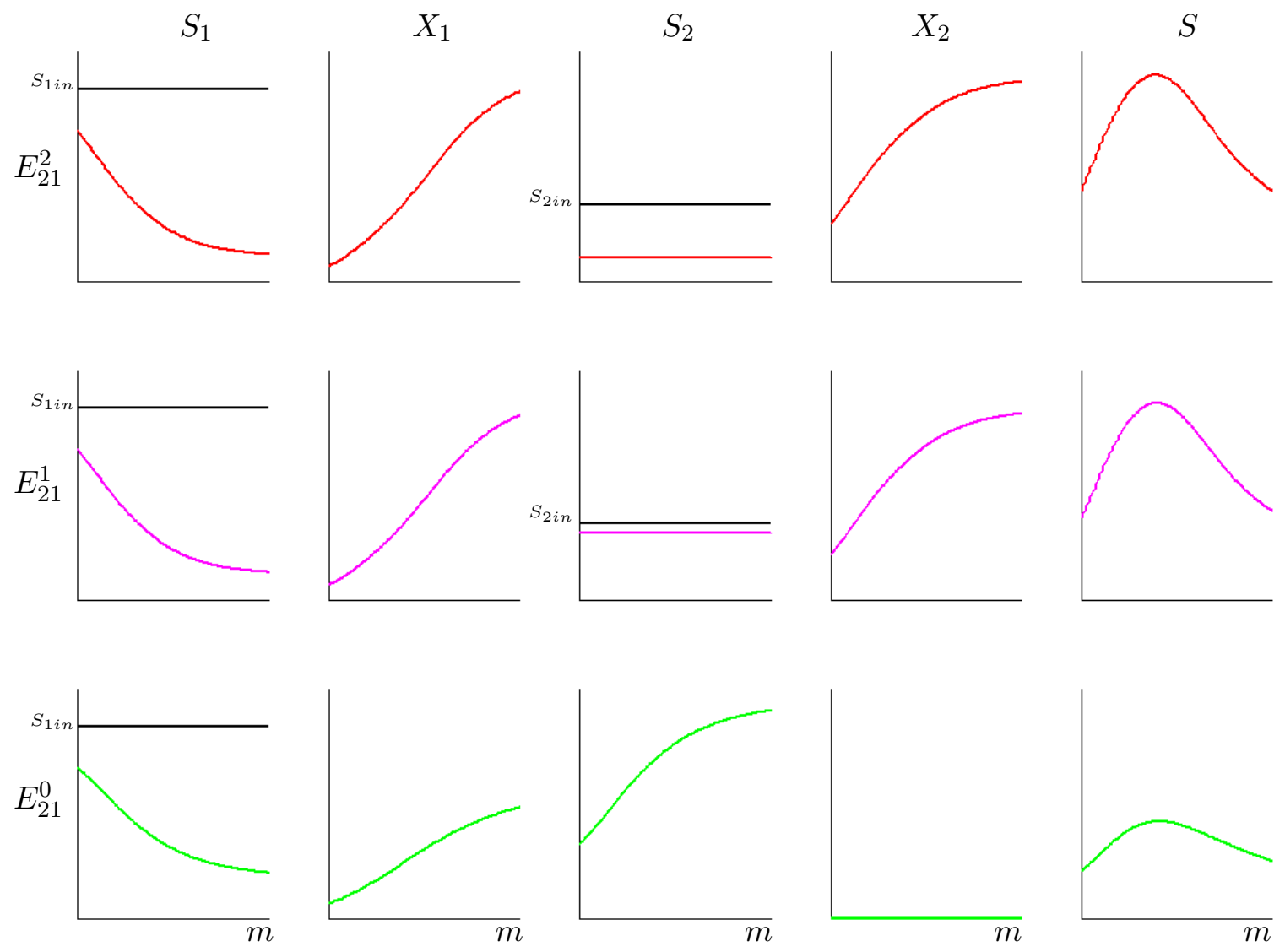

Figure 9: The components of the equilibrium points $E_{21}^{0}$ (in green), $E_{21}^{1}$ (in red) and $E_{21}^{2}$ (in magenta) in the case A. 
$E_{1}^{2}$, which do not depend on $m$ and which exist if and only if (21) holds. If the value of $m$ increases continuously, then the graph of $F_{m}(S)$ intersects first with the graphs of $H_{1}\left(S_{1}\right)$, then with the graph of $H_{2}\left(S_{1}\right)$ and finally with the graph of $G\left(S_{1}\right)$, in each case leading to the apparition of new equilibria. Let us use the following notations for the bifurcation values of $m$ as it is illustrated in Fig. 10:

- $m=m_{c 1}$ : the value of $m$ for the first intersection of $F_{m}$ with $H_{1}$,

- $m=m_{c 2}$ : the value of $m$ for the second intersection of $F_{m}$ with $H_{2}$,

- $m=m_{c 3}$ : the value of $m$ for the third intersection of $F_{m}$ with $G$,

- $m=m_{c 4}$ : the value of $m$ beyond which we have $S_{1 i n}=S_{1}^{*}$ for $E_{22}^{1}$ (the condition (26) becomes not satisfied and consequently $E_{22}^{1}$ passes to the gray area),

- $m=m_{c 5}$ : the value of $m$ beyond which we have $S_{1 i n}=S_{1}^{*}$ for $E_{22}^{2}$ (the condition (26) becomes not satisfied and consequently $E_{22}^{2}$ passes to the gray area).

Each time when the value of $m$ exceeds $m_{c i}, i=1 . .5$, we have equilibria bifurcation. Thus, the system can have new equilibria or loose equilibria. More precisely, in Table 3 we give equilibria and their nature according to $m$, where $\mathrm{T}$ stands for Trivial Equilibria, $F_{m} \cap H_{1}, F_{m} \cap H_{2}$ and $F_{m} \cap G$ stand for Equilibria obtained by the intersections of the graph $F_{m}$ with graphs $H_{1}, H_{2}$ and $G$, respectively, and $\mathrm{S}$ and U stand for a Stable and an Unstable Equilibrium, respectively. If there is no symbol, then it means that the equilibrium does not exist. 


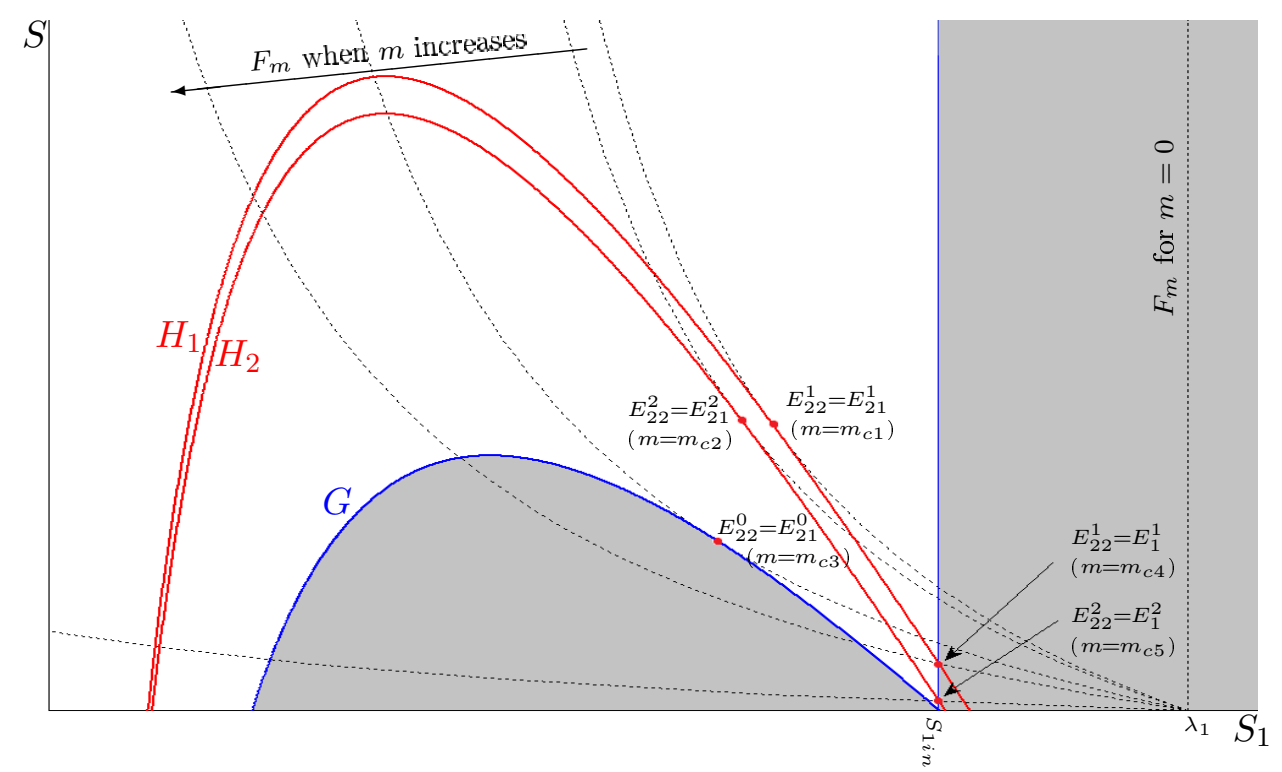

Figure 10: Values $m_{c i}, i=1 . .5$ of $m$ giving equilibria bifurcation in the generic case B.

Table 3: Equilibria and their nature in the generic case B represented in Fig. 6.

\begin{tabular}{|c|c|c|c|c|c|c|c|c|c|c|}
\hline \multirow{3}{*}{ case $B$} & \multirow{3}{*}{ Condition } & \multicolumn{9}{|c|}{ Equilibria and nature } \\
\hline & & \multicolumn{3}{|c|}{$\mathrm{T}$} & \multicolumn{2}{|c|}{$F \cap G$} & \multicolumn{2}{|c|}{$F \cap H_{1}$} & \multicolumn{2}{|c|}{$F \cap H_{2}$} \\
\hline & & $E_{0}^{0}$ & $E_{1}^{1}$ & $E_{1}^{2}$ & $E_{21}^{0}$ & $E_{22}^{0}$ & $E_{21}^{1}$ & $E_{22}^{1}$ & $E_{21}^{2}$ & $E_{22}^{2}$ \\
\hline B.1 & $0 \leq m<m_{c 1}$ & $\mathrm{~S}$ & $\mathrm{~S}$ & $\mathrm{U}$ & & & & & & \\
\hline B.2 & $m_{c 1}<m<m_{c 2}$ & $\mathrm{~S}$ & $\mathrm{~S}$ & $\mathrm{U}$ & & & $\mathrm{S}$ & $\mathrm{U}$ & & \\
\hline B.3 & $m_{c 2}<m<m_{c 3}$ & $\mathrm{~S}$ & $\mathrm{~S}$ & $\mathrm{U}$ & & & $\mathrm{S}$ & $\mathrm{U}$ & $\mathrm{U}$ & $\mathrm{U}$ \\
\hline B.4 & $m_{c 3}<m<m_{c 4}$ & $\mathrm{~S}$ & $\mathrm{~S}$ & $\mathrm{U}$ & $\mathrm{S}$ & $\mathrm{U}$ & $\mathrm{S}$ & $\mathrm{U}$ & $\mathrm{U}$ & $\mathrm{U}$ \\
\hline $\mathrm{B} .5$ & $m_{c 4}<m<m_{c 5}$ & $\mathrm{~S}$ & $\mathrm{U}$ & $\mathrm{U}$ & $\mathrm{S}$ & $\mathrm{U}$ & $\mathrm{S}$ & & $\mathrm{U}$ & $\mathrm{U}$ \\
\hline B.6 & $m_{c 5}<m$ & $\mathrm{~S}$ & $\mathrm{U}$ & $\mathrm{U}$ & $\mathrm{S}$ & $\mathrm{U}$ & $\mathrm{S}$ & & $\mathrm{U}$ & \\
\hline
\end{tabular}

Here, with the parameter values used, the condition (21) is satisfied (see (32)). Thus, the system has generic bistability behavior if there is no intersection between $F_{m}$ and $H_{i}, i=1,2$ and $G$ as illustrated in case B.1 of Table 3. In case B.2, the system can have five equilibria of which three are 
locally stable (tristability behavior). In case B.3, we have seven equilibria, such that $E_{21}^{2}$ and $E_{22}^{2}$, obtained from the intersection of $F_{m}$ with $H_{2}$, are both unstable. Under some operating conditions in case B.4, the system can have up to nine equilibria after the graph $F_{m}$ intersects with the graph $G$. In this case, four equilibria are stable and the system exhibits quadristability behavior.

For some increasing values of $m$, the system can loose some equilibria. There are cases B.5 and B. 6 of Table 3, when $E_{22}^{1}$ and $E_{22}^{2}$, respectively, pass into the gray area because henceforth condition (26) is not satisfied.

On the contrary to trivial equilibria $E_{0}^{0}, E_{1}^{1}$ and $E_{1}^{2}$, which do not depend on $m$, equilibria $E_{2 i}^{0}, E_{2 i}^{2}$ and $E_{2 i}^{1}, i=1,2$ of Fig. 11 depend on $m$ and can undergo bifurcation. For instance, we have the first bifurcation of $E_{2 i}^{1}$ for $m=m_{c 1}$. However, the equilibrium $E_{22}^{1}$ disappears later on for $m \geq m_{c 4}$, because $S_{1}^{*}$ becomes higher than $S_{1 i n}$. Equilibria $E_{2 i}^{2}$ and $E_{2 i}^{0}$ appear for $m=m_{c 2}$ and $m=m_{c 3}$ respectively.

In generic case B, the system (7)-(11) thus exhibits a rich qualitative behavior when the value of the bifurcation parameter $m$ is changed. In particular, under some operating conditions (for $0<m<m_{c 1}$ ), the system AM2b (7)-(11) behaves exactly as the original system AM2 [2] and functions in bistability. However, under other operating conditions, when the value of $m$ changes the system AM2b can have nine equilibria (for $m_{c 3}<m<m_{c 4}$ ) and functions in quadri-stability while for other values of $m$, it can function in tristability. 
$S_{1}$
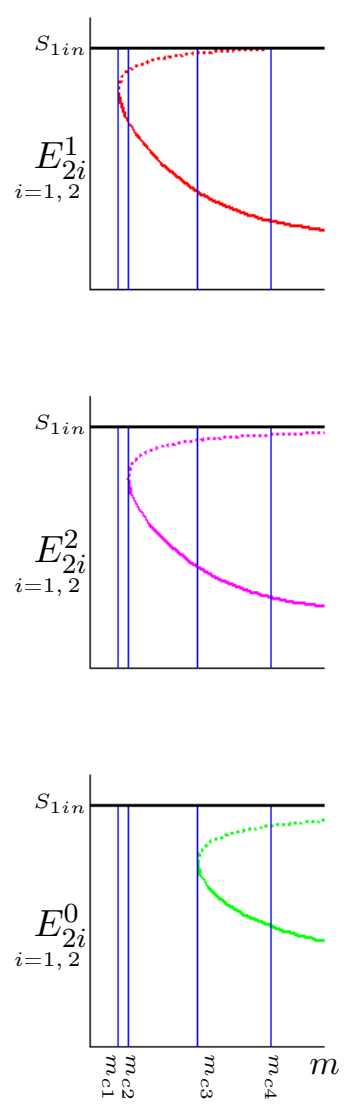

$X_{1}$
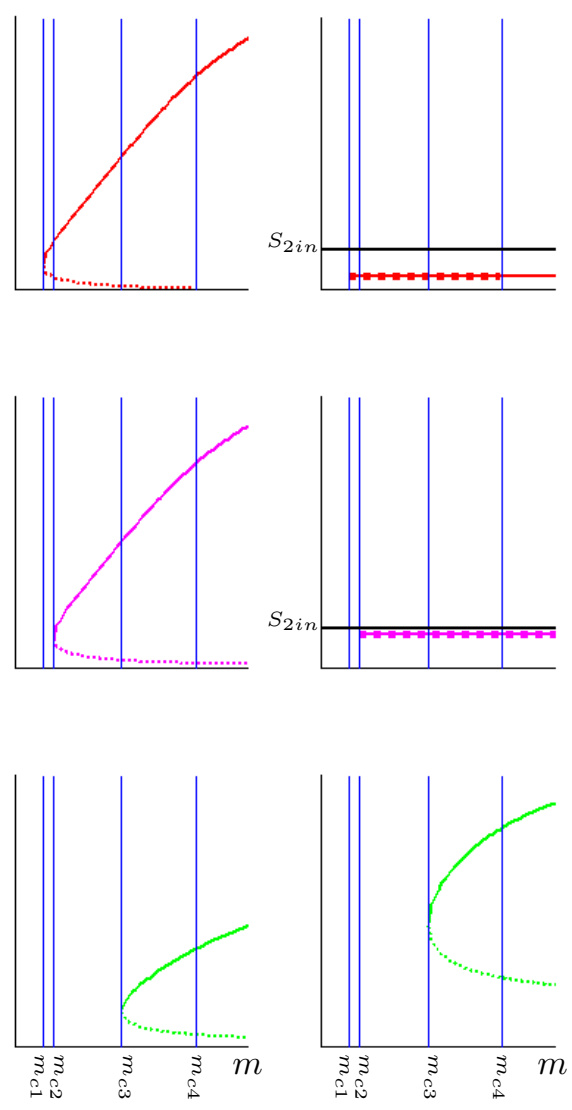

$S_{2}$
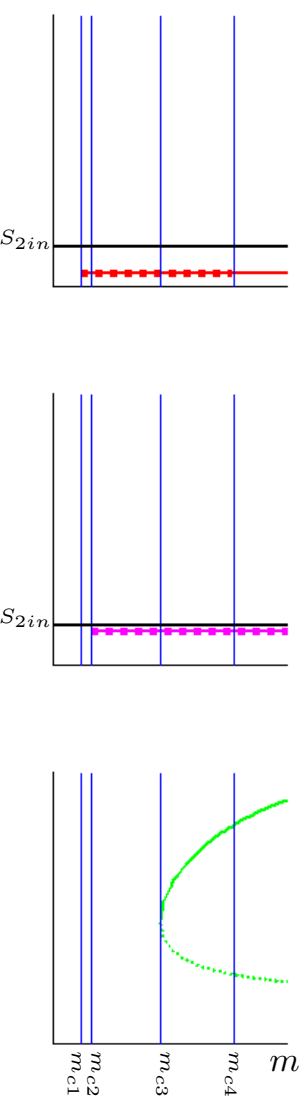

$X_{2}$

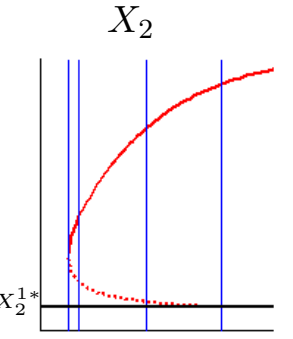

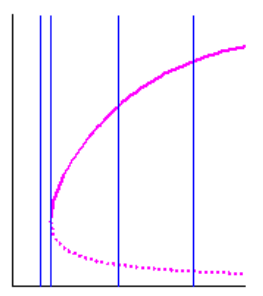

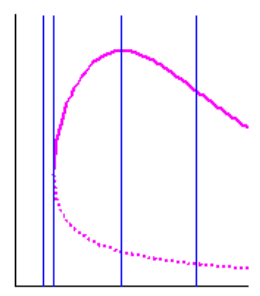

$S$
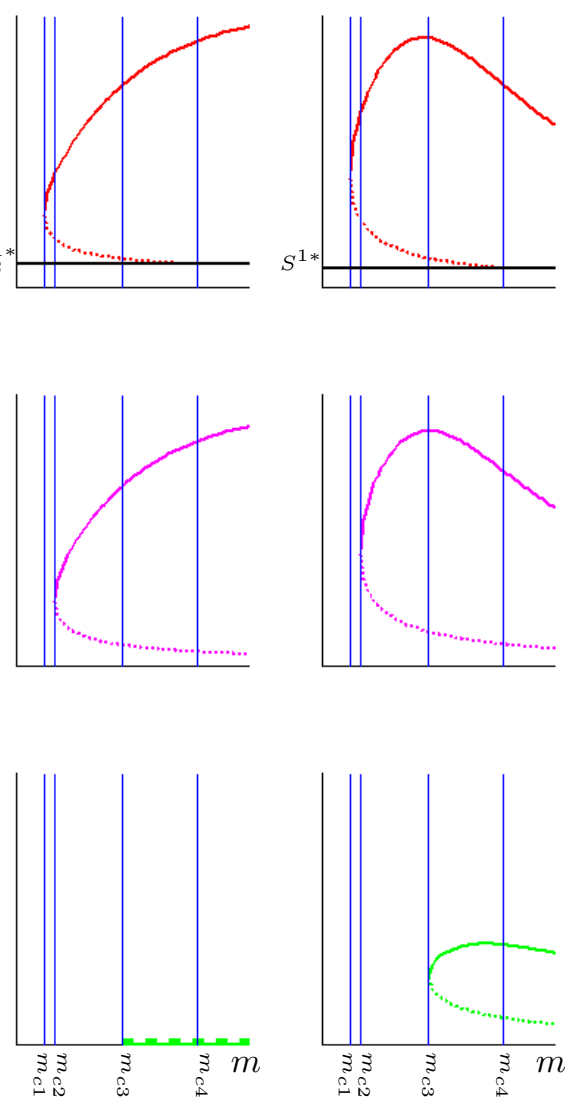

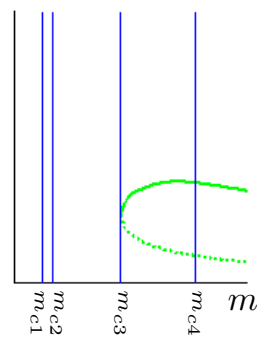

Figure 11: The components of the equilibrium points $E_{21}^{1}$ (in solid red), $E_{22}^{1}$ (in dash red), $E_{21}^{2}$ (in solid magenta), $E_{22}^{2}$ (in dash magenta), $E_{21}^{0}$ (in solid green) and $E_{22}^{0}$ (in dash green) in case B. The vertical lines correspond to the bifurcation values of $m_{c 1}, m_{c 2}, m_{c 3}$ and $m_{c 4}$. The bifurcation value $m_{c 5}$, for which the component $S_{1}^{*}$ of $E_{22}^{2}$ is equal to $S_{1 i n}$, is too large and is not shown on the figure. 


\subsection{Generic Case C: Equilibria bifurcation for the AM2b, second example}

To illustrate the richness of the possible qualitative behavior of the AM2b model, we present in this section the generic case $\mathrm{C}$, which is is described by Fig. 7. As shown previously in the generic case B, the system can have only the washout equilibira for $m=0$, because there is no intersection between $F_{m}(S), G\left(S_{1}\right)$ and $H_{i}\left(S_{1}\right), i=1,2$. Washout equilibria exist if and only if condition (21) is verified. Here, in this generic case, we have:

$$
S_{2}^{1 *}=0.0 .1652<S_{2 i n}=0.6<S_{2}^{2 *}=1.6348
$$

The equilibrium does not exist since $S_{2}^{2 *}>S_{2 i n}$. The system has two washout equilibria $E_{0}^{0}$ and $E_{1}^{1}$. If the value of $m$ increases, then we have intersections between $F_{m}(S)$ and $G\left(S_{1}\right)$ and $H_{i}\left(S_{1}\right), i=1,2$ and, thus, equilibria bifurcation are obtained as illustrated in Fig. 12.

Beyond $m=m_{c 4}$ (see Fig. 12), there is a large bifurcation, where $H_{2}\left(S_{1}^{*}\right)<G\left(S_{1}^{*}\right)$ for $E_{22}^{2}$. Henceforth, the condition (31) is not satisfied and the equilibrium $E_{22}^{2}$ disappears in the gray area. The equilibria and their nature are given in Table 4 . The system can have two equilibria for $m<m_{c 1}$ (see case C.1). Later on, it acquires new equilibria for $m_{c 1}<m<m_{c 4}$ (see cases C.2-C.4), or losses equilibria for $m>m_{c 4}$ (see cases C.5-C.6). Notice that in case C.4, it has eight equilibria and functions in tristability $\left(E_{1}^{1}, E_{21}^{0}\right.$ and $E_{21}^{1}$ are locally stable).

In Fig. 13, we present equilibria $E_{2 j}^{0}, E_{2 j}^{1}$ and $E_{2 j}^{2}, i=1,2$. 


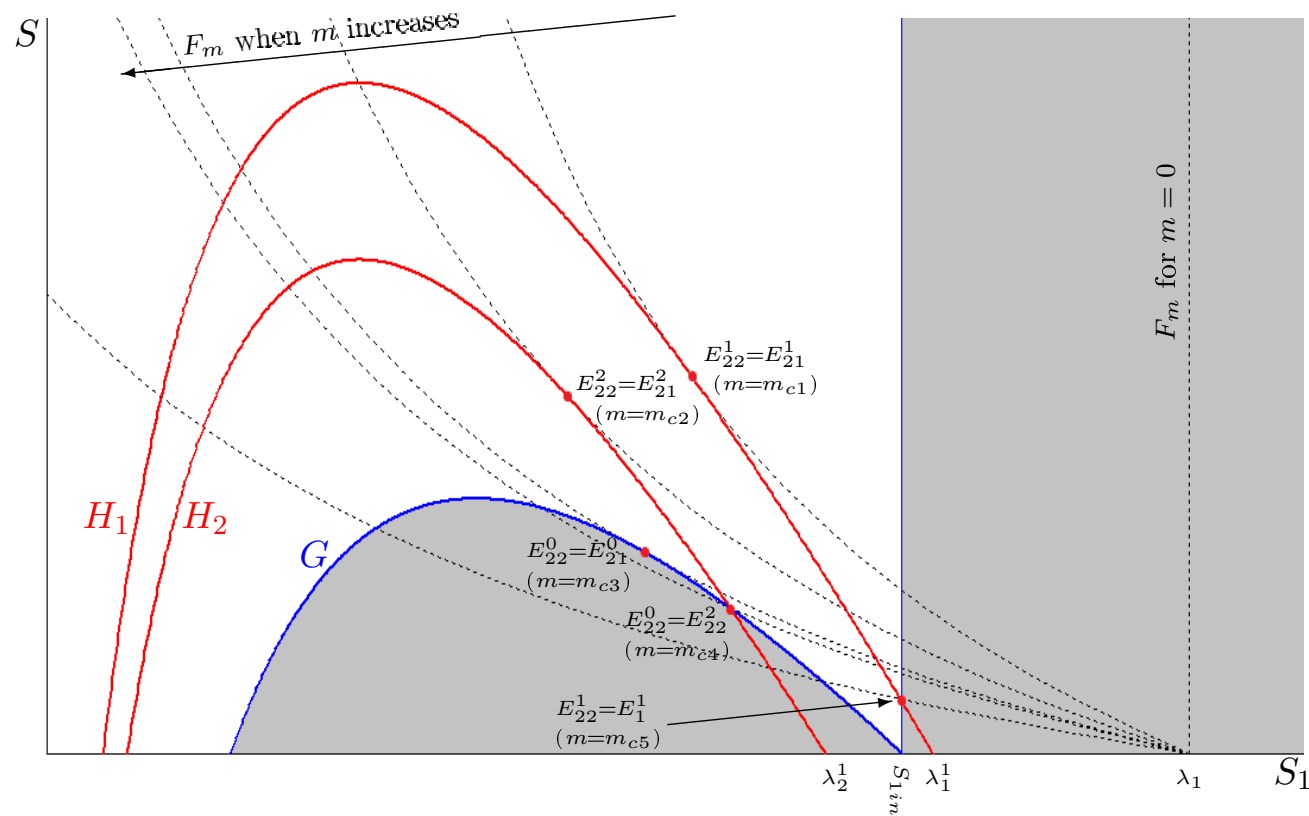

Figure 12: Values $m_{c i}, i=1 . .5$ of $m$ giving equilibria bifurcation in the generic case $\mathrm{C}$.

Table 4: Equilibria and their nature in generic case $\mathrm{C}$ represented in Fig. 7. $S$ and $U$ have the same signification as in Table 3.

\begin{tabular}{|c|c||c|c|c|c|c|c|c|c|}
\hline \multirow{2}{*}{ case C } & \multirow{2}{*}{ Condition } & \multicolumn{7}{|c|}{ Equilibria and nature } \\
\cline { 3 - 10 } & & \multicolumn{2}{|c|}{$\mathrm{T}$} & \multicolumn{2}{|c|}{$F \cap G$} & \multicolumn{2}{c|}{$F \cap H_{1}$} & \multicolumn{3}{|c|}{$F \cap H_{2}$} \\
\hline & & $E_{0}^{0}$ & $E_{1}^{1}$ & $E_{21}^{0}$ & $E_{22}^{0}$ & $E_{21}^{1}$ & $E_{22}^{1}$ & $E_{21}^{2}$ & $E_{22}^{2}$ \\
\hline \hline $\mathbf{C . 1}$ & $0 \leq m<m_{c 1}$ & $\mathrm{U}$ & $\mathrm{S}$ & & & & & & \\
\hline $\mathbf{C . 2}$ & $m_{c 1}<m<m_{c 2}$ & $\mathrm{U}$ & $\mathrm{S}$ & & & $\mathrm{S}$ & $\mathrm{U}$ & & \\
\hline $\mathbf{C . 3}$ & $m_{c 2}<m<m_{c 3}$ & $\mathrm{U}$ & $\mathrm{S}$ & & & $\mathrm{S}$ & $\mathrm{U}$ & $\mathrm{U}$ & $\mathrm{U}$ \\
\hline $\mathbf{C . 4}$ & $m_{c 3}<m<m_{c 4}$ & $\mathrm{U}$ & $\mathrm{S}$ & $\mathrm{S}$ & $\mathrm{U}$ & $\mathrm{S}$ & $\mathrm{U}$ & $\mathrm{U}$ & $\mathrm{U}$ \\
\hline $\mathbf{C . 5}$ & $m_{c 4}<m<m_{c 5}$ & $\mathrm{U}$ & $\mathrm{S}$ & $\mathrm{S}$ & $\mathrm{U}$ & $\mathrm{S}$ & $\mathrm{U}$ & $\mathrm{U}$ & \\
\hline $\mathbf{C . 6}$ & $m_{c 5}<m$ & $\mathrm{U}$ & $\mathrm{U}$ & $\mathrm{S}$ & $\mathrm{U}$ & $\mathrm{S}$ & & $\mathrm{U}$ & \\
\hline
\end{tabular}

\subsection{Qualitative difference between AM2 and AM2b models}

We have shown that models AM2 and AM2b may show significantly different qualitative behavior depending on model parameters and operating 


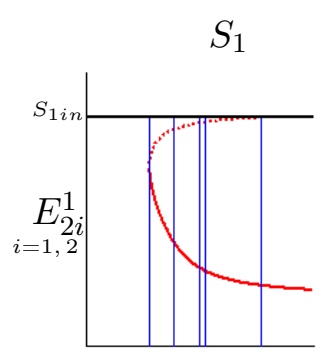

$X_{1}$
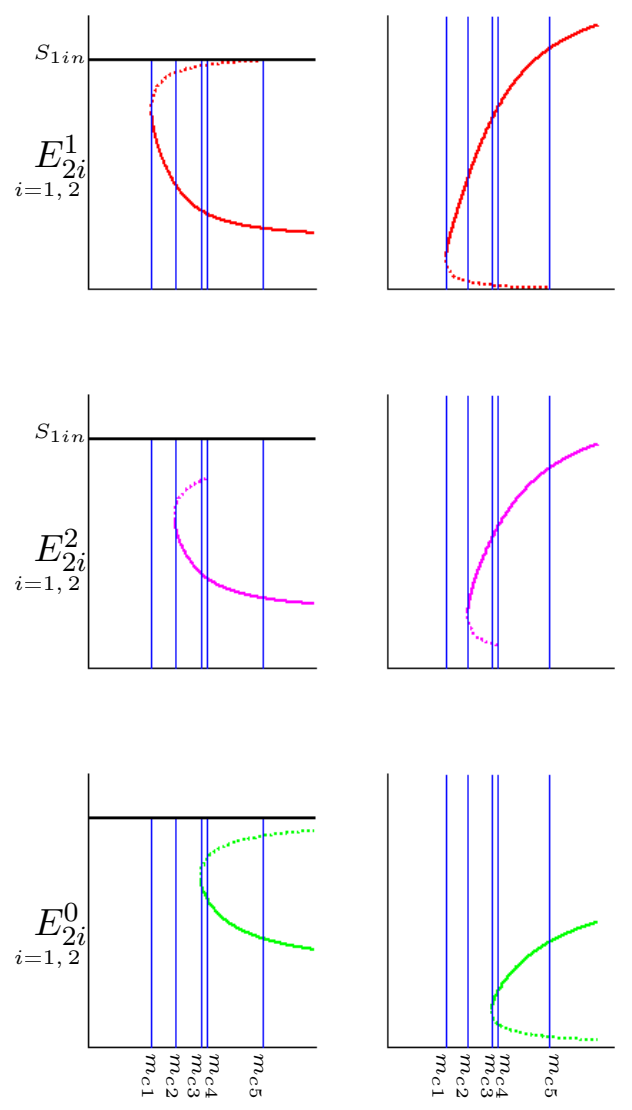

$S_{2}$
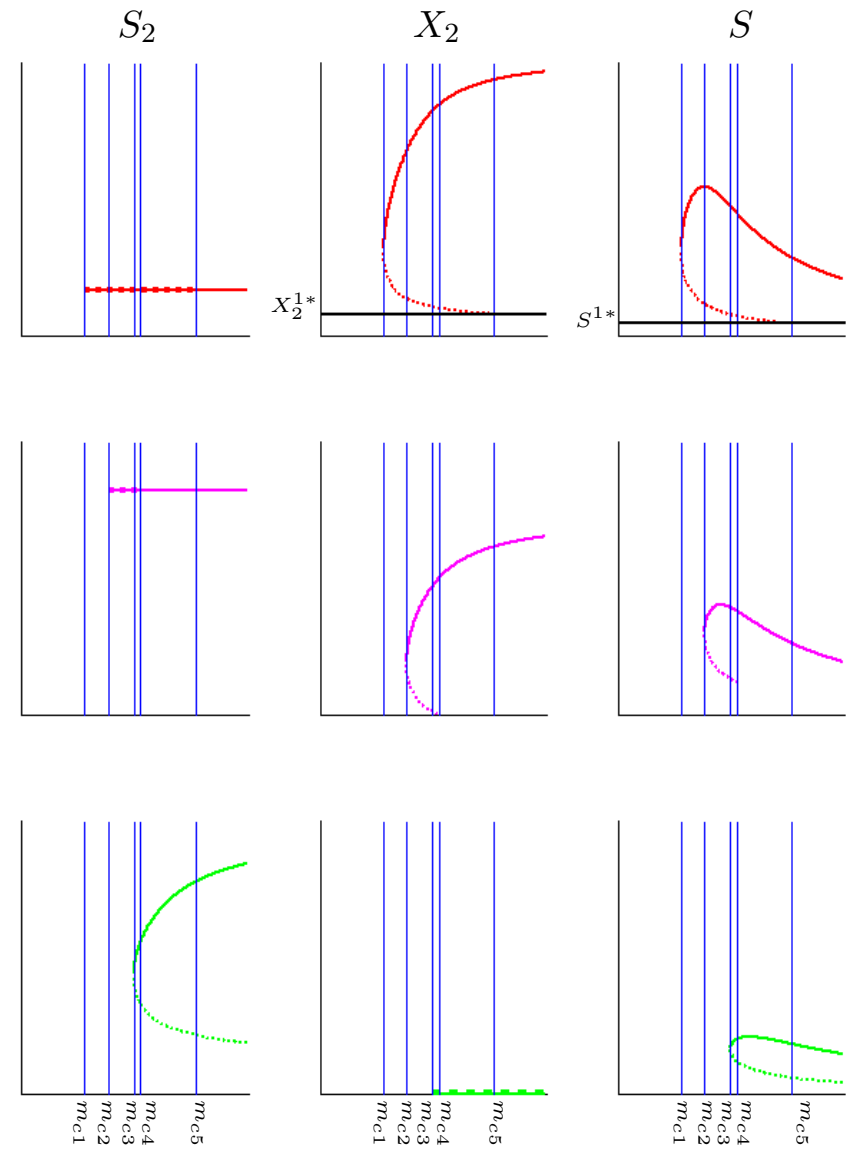

Figure 13: The components of the equilibrium points $E_{21}^{1}$ (in solid red), $E_{22}^{1}$ (in dash red), $E_{21}^{2}$ (in solid magenta), $E_{22}^{2}$ (in dash magenta), $E_{21}^{0}$ (in solid green) and $E_{22}^{0}$ (in dash green) in case $\mathrm{C}$. The vertical lines correspond to the bifurcation values of $m_{c 1}, m_{c 2}, m_{c 3}$, $m_{c 4}$ and $m_{c 5}$. 
conditions. In particular, the steady-state value of the substrate concentration $S_{1}$ (denoted $S_{1}^{*}$ ) is not affected by the input substrate concentration $S_{1 \text { in }}$ in the AM2 or, to put it differently, in the AM2b with $m=0$. When $m$ differs from 0 , it should be noted that the steady-state value of $S_{1}$ changes with $S_{1 i n}$. More precisely, $S_{1}^{*}$ is a decreasing function of $S_{1 \text { in }}$ (cf. Fig. 14, the intersection between curves $F_{m}$ and $H_{1}$ when $S_{1 i n}$ increases and Fig. 15).

This important qualitative difference between the AM2 and the AM2b models can be used for modeling purposes in order to validate this new model in the following way: assuming we have access to steady-state data, we need only to look at the different steady-states obtained with different $S_{1 \text { in }}$ and compare the corresponding values of substrates $S_{1}^{*}$. If they differ, AM2 is not a good candidate for modeling while AM2b can be used instead.

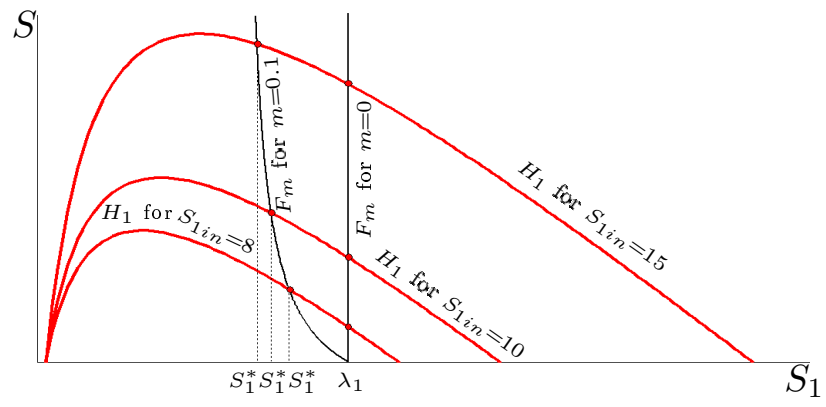

Figure 14: Modeling of the equilibrium $S_{1}^{*}$ with the model AM2b when $S_{1 \text { in }}$ changes. Here, $K_{1}=10, S_{2}=0.6, D_{1}=0.25, m=0-1$ and $S_{1 \text { in }}=8-10-15$. Other parameters values are those given in Table 1 .

\section{Conclusion}

In this paper, we have developed and studied a mathematical model for an AnMBR. The new model, named AM2b, was developed by modifying the 


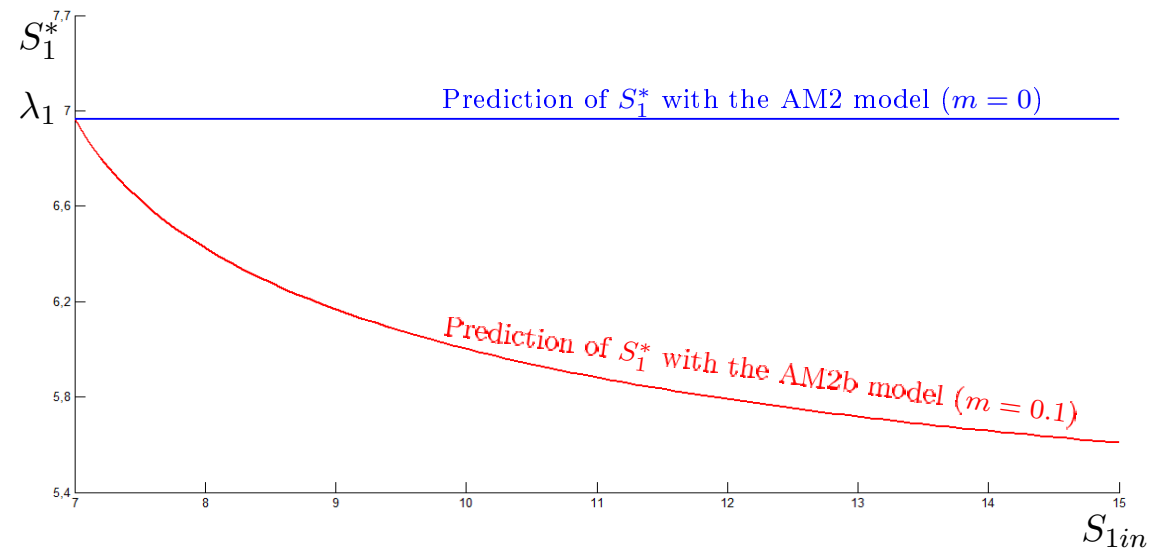

Figure 15: Evolution of the equilibrium $S_{1}^{*}$ according to $S_{1 \text { in }}$ for $m=0$ and $m=0.1$. Parameter values used are the same as in Fig. 14.

AM2 model [1] to integrate SMP production and degradation. The mass balance equations are proposed in considering a new reaction network taking into account certain assumptions about the functioning of the AnMBR, in particular the behavior of the different variables with respect to the presence of a membrane. A graph-based approach to study the developed model has been presented and illustrated by numerical simulations. We studied three generic cases of system behavior in the light of the values of the relevant biological parameters, in particular the maximum growth rate $m$ of acidogenic bacteria on SMP. We show that in one of these cases, the AM2b system behaves exactly like the AM2 system. It always operates in bistability for all value of $m$. However, in the two other generic cases, the system displays rich qualitative behavior and can have up to nine equilibria while showing multi-stability behavior. We also show that the AM2b system can predict the change of equilibrium $S_{1}^{*}$ of the organic matter when the influent concentration $S_{1 i n}$ changes. 
Perspectives for further work include: i) the design of observers to estimate biomasses $X_{1}$ and $X_{2}$, SMP and kinetics; ii) the validation of hypotheses to confront the AM2b model with experimental data; and iii) the coupling of this model with models of membrane modules in order to design hybrid models for the complete description of an AnMBR for use in process control. 


\begin{tabular}{|c|c|c|}
\hline \multicolumn{3}{|c|}{ Abbreviations } \\
\hline$M B R$ & Membrane BioReactor & \\
\hline$A n M B R$ & Anaerobic Membrane BioReactor & \\
\hline$S M P$ & Soluble Microbial Products & \\
\hline$E P S$ & Extra-cellular Polymeric Substances & \\
\hline$U A P$ & Utilization Associated Products & \\
\hline$B A P$ & Biomass Associated Products & \\
\hline$W W T P$ & Waste Water Treatment Plant & \\
\hline$A S M$ & Activated Sludge Model & \\
\hline$A M 2$ & 2-steps Acidogenesis - Methanogenesis model & \\
\hline$A M 2 b$ & AM2 model with SMP dynamics & \\
\hline$C O D$ & Chemical Oxygen Demand & \\
\hline$V F A$ & Volatile Fatty Acids & \\
\hline \multicolumn{3}{|c|}{ Nomenclature } \\
\hline$X_{1}$ & concentration of acidogenic bacteria & {$[g / l]$} \\
\hline$X_{2}$ & concentration of methanogenic bacteria & {$[g / l]$} \\
\hline$S_{1}$ & concentration of organic matter & {$[g / l]$} \\
\hline$S_{2}$ & concentration of VFA & {$[g / l]$} \\
\hline$S_{1 i n}$ & the input concentration of $S_{1}$ & {$[g / l]$} \\
\hline$S_{2 i n}$ & the input concentration of $S_{2}$ & {$[g / l]$} \\
\hline$S$ & concentration of SMP & {$[g / l]$} \\
\hline $\mathrm{CH}_{4}$ & produced methane & \\
\hline $\mathrm{CO}_{2}$ & carbon dioxide & \\
\hline$k_{1}$ & yield of $S_{1}$ degradation by $X_{1}$ & {$\left[g C O D_{X_{1}} / g C O D_{S_{1}}\right]$} \\
\hline$k_{2}$ & yield of $S_{2}$ production from $S_{1}$ & {$\left[g C O D_{S_{2}} / g C O D_{S_{1}}\right]$} \\
\hline$k_{3}$ & yield of $S_{2}$ degradation by $X_{2}$ & {$\left[g C O D_{X_{2}} / g C O D_{S_{2}}\right]$} \\
\hline$k_{4}$ & yield of $C O_{2}$ production from $S_{1}$ & {$\left[g C O D_{X_{1}} / g C O D_{S_{1}}\right]$} \\
\hline$k_{5}$ & yield of $\mathrm{CO}_{2}$ production from $S_{2}$ & {$\left[g C O D_{\mathrm{CO}_{2}} / g C O D_{S_{2}}\right]$} \\
\hline$k_{6}$ & yield of $\mathrm{CH}_{4}$ production from $S_{2}$ & {$\left[g C O D_{\mathrm{CH}_{4}} / g C O D_{S_{2}}\right]$} \\
\hline$b_{1}$ & yield of $S M P$ degradation by $X_{1}$ & {$\left[g C O D_{X_{1}} / g C O D_{S M P}\right]$} \\
\hline$b_{2}$ & yield of $S_{2}$ production from $S M P$ & {$\left[g C O D_{S_{2}} / g C O D_{S M P}\right]$} \\
\hline$b_{3}$ & yield of $S M P$ production from $S_{1}$ & {$\left[g C O D_{S M P} / g C O D_{S_{1}}\right]$} \\
\hline$b_{4}$ & yield of $S M P$ production from $S_{2}$ & {$\left[g C O D_{S M P} / g C O D_{S_{2}}\right]$} \\
\hline$\beta$ & SMP fraction leaving the bioreactor & \\
\hline$\Delta P$ & transmembrane pressure & {$[P a]$} \\
\hline$\Delta P_{c r i}$ & critic transmembrane pressure & {$[P a]$} \\
\hline$J$ & membrane flux & {$\left[L /\left(h \cdot m^{2}\right)\right]$} \\
\hline$J_{\text {limite }}$ & threshold membrane flux & {$\left[L /\left(h . m^{2}\right)\right]$} \\
\hline$J_{c r i}$ & critic membrane flux & {$\left[L /\left(h . m^{2}\right)\right]$} \\
\hline
\end{tabular}




\begin{tabular}{|lll|}
\hline$\mu_{1}$ & acidogenesis kinetics & {$[1 / d]$} \\
$\mu_{2}$ & methanogenesis kinetics & {$[1 / d]$} \\
$\mu$ & kinetic of SMP degradation & {$[1 / d]$} \\
$m_{1}$ & maximum acidogenic biomass growth rate on $S_{1}$ & {$[1 / d]$} \\
$m_{2}$ & maximum methanogenic biomass growth rate on $S_{2}$ & {$[1 / d]$} \\
$m$ & maximum acidogenic biomass growth rate on $S$ & {$[1 / d]$} \\
$K_{1}$ & half-saturation constant associated with $S_{1}$ & {$[g / L]$} \\
$K_{2}$ & half-saturation constant associated with $S_{2}$ & {$[g / L]$} \\
$K_{i}$ & inhibition constant associated with $S_{2}$ & {$[g / L]$} \\
$K$ & half-saturation constant associated with $S$ & {$[g / L]$} \\
$Q_{i n}$ & the input flow of the bioreactor & {$[L / d]$} \\
$Q_{o u t}$ & the output flow of the bioreactor & {$[L / d]$} \\
$Q_{1}$ & the withdraw flow from the bioreactor & {$[L / d]$} \\
$V$ & the volume of the bioreactor & {$[L]$} \\
$D$ & the dilution rate & {$[1 / d]$} \\
$D_{0}$ & the decay rate of biomasses & {$[1 / d]$} \\
$D_{1}$ & the biomass withdraw & {$[1 / d]$} \\
$\xi^{*}$ & the value of the component $\xi$ at equilibrium $\left(\xi=S_{1}, X_{1}, S_{2}, X_{1}\right.$ or $\left.S\right)$ & \\
$E^{*}, E_{2 i}^{j}$ & equilibria, $i=1,2, j=0,1,2$ &
\end{tabular}




\section{References}

[1] Bernard, O., and Hadj-Sadock, Z., and Dochain, D., and Genovesi, A. and Steyer, J.P. Dynamical Model Development and Parameter Identification for an Anareobic Wastewater Treatment Process. Biotechnology and Bioengineering, 75:424-438, 2001.

[2] Benyahia, B., and Sari, T., and Cherki, B, and Harmand, J. Bifurcation and stability analysis of a two step model for monitoring anaerobic digestion processes. Journal of Process Control, 22:1008-1019, 2012.

[3] B. Benyahia, T. Sari, J. Harmand, B. Cherki. Modeling of the Soluble Microbial Products (SMP) in Anaerobic Membrane BioReactors (AMBR): Equilibria ans stability of the AM2b model. Proceeding of 18th IFAC Word Congress, Milano, Italy, pp. 3789-3794, 2011.

[4] Ng, A.N.L. and Kim, A.S. A mini-review of modeling studies on membrane bioreactor (MBR) treatment for municipal wastewaters. Desalination, 212:261-281, 2007.

[5] Barker, D.J. and Stuckey, D.C. A review of soluble microbial products (SMP) in wastewater treatment systems. Water Ressources, 33(14):3063-3082, 1999.

[6] Luedeking, R. and Piret, E.C. A kinetic study of lactic acid fermentation batch process at controlled pH. Journal of Biochem. Microbiol. Tech. Eng., 1(4):393-412, 1959.

[7] Noguera, D.R., Araki, N. and Rittmann, B.E. Soluble Microbial 
Products (SMP) in Anaerobic Chemostats. Biotechnol. Bioeng., 44(9):1040-7, 1994.

[8] Barker, D.J. and Stuckey, D.C. Modelling of soluble Microbial Products in Anaerobic Digestion: the effect of Feed Strength and Composition. Water Environ. Res., 73(2):173-184, 2001.

[9] Laspidou, C.S. and Rittmann, B.E. A unified theory for extracellular polymeric substances, soluble microbial products, and active and inert biomass. Water Res., 36:2711-2720, 2002.

[10] Aquino, S.F. and Stuckey, D.C. Integrated model of the production of soluble microbial products (SMP) and extracellular polymeric substances (EPS) in anaerobic chemostats during transient conditions. Biochemical Engineering Journal, 38:138-146, 2008.

[11] Sbarciog, M., and Loccufier, M. and Noldus, E. Determination of appropriate operating strategies for anaerobic digestion systems. Biochemical Engineering Journal, 51:180-188, 2010. 